\title{
A PROOF OF CONCEPT FOR CROWDSOURCING COLOR PERCEPTION EXPERIMENTS
}

\author{
A Thesis \\ presented to \\ the Faculty of California Polytechnic State University \\ San Luis Obispo
}

\author{
In Partial Fulfillment \\ of the Requirements for the Degree \\ Master of Science in Computer Science
}

by

Ryan McLeod

June 2014 
(C) 2014

Ryan McLeod

ALL RIGHTS RESERVED 


\section{COMMITTEE MEMBERSHIP}

TITLE:

A Proof of Concept for Crowdsourcing Color Perception Experiments

AUTHOR:

Ryan McLeod

DATE SUBMITTED: June 2014

COMMITTEE CHAIR: Associate Professor John Bellardo, Ph.D. Department of Computer Science

COMMITTEE MEMBER: Professor Franz Kurfess, Ph.D. Department of Computer Science

COMMITTEE MEMBER: Assoc iate Professor Brian Lawler, Department of Graphic Communications 


\begin{abstract}
A Proof of Concept for Crowdsourcing Color Perception Experiments

Ryan McLeod
\end{abstract}

Accurately quantifying the human perception of color is an unsolved problem. There are dozens of numerical systems for quantifying colors and how we as humans perceive them, but as a whole, they are far from perfect. The ability to accurately measure color for reproduction and verification is critical to industries that work with textiles, paints, food and beverages, displays, and media compression algorithms. Because the science of color deals with the body, mind, and the subjective study of perception, building models of color requires largely empirical data over pure analytical science. Much of this data is extremely dated, from small and/or homogeneous data sets, and is hard to compare. While these studies have somewhat advanced our understanding of color adequately, making significant, further progress without improved datasets has proven difficult if not impossible. I propose new methods of crowdsourcing color experiments through color-accurate mobile devices to help develop a massive, global set of color perception data to aid in creating a more accurate model of human color perception. 


\section{ACKNOWLEDGMENTS}

Thanks to:

- Dr. Bellardo, for inspiring this project through his iOS class and for believing in me when everyone else thought I was just putting crayons up my nose.

- Gary Hughes, for his time, kindness, and statistics.

- Steve Upton, for his clarifications and metaphors.

- Mark Meyer, for his explanations, images, and encouragement.

- Jeff Yurek, for the permission to use some of his great graphics.

- The Cal Poly Computer Science Department.

- All of the libraries that let me excessively borrow Mr. Kuehni's book.

- My family for encouraging me to pursue the Master's program.

- Everyone who has let me wave my arms and explain color to them, has inspired me, and has pushed me to keep on researching and learning. 


\section{TABLE OF CONTENTS}

List of Tables viii

List of Figures ix

1 Introduction 1

1.1 Problematic Perception . . . . . . . . . . . . . . . . . . 4

1.2 Limited Data . . . . . . . . . . . . . . . . . . 5

1.3 Crowdsourcing Solution $\ldots \ldots \ldots \ldots$

$\begin{array}{lll}2 & \text { Background } & 12\end{array}$

$2.1 \quad$ Color . . . . . . . . . . . . . . . . . . . . . . . . . . . . . . . . . . . 12

2.2 Modeling and Ordering Color . . . . . . . . . . . . . 13

2.3 Perceptual Uniformity $\ldots \ldots \ldots \ldots$

2.4 Established color spaces $\ldots \ldots \ldots \ldots$

$2.4 .1 \quad \mathrm{RGB} \ldots \ldots \ldots \ldots \ldots \ldots$

$2.4 .2 \quad \mathrm{HSV} \& \mathrm{HSL} \ldots \ldots \ldots \ldots \ldots$

$2.4 .3 \quad$ CIE XYZ . . . . . . . . . . . . . . . . . . . . . . . 19

2.4 .4 CIE Lab . . . . . . . . . . . . . . . . . . . . . 23

2.5 Color Atlases $\ldots \ldots \ldots \ldots$

2.5 .1 Munsell . . . . . . . . . . . . . . . . . . 26

2.5.2 Optical Society of America Uniform Color Scales (OSA-UCS) 27

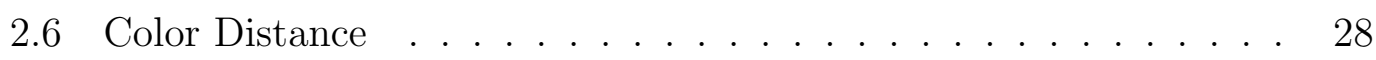

2.6 .1 The Curent State of The Art: CIEDE2000 . . . . . . . 29

3 A New Way to Test 32

$3.1 \quad$ We Require Additional Data . . . . . . . . . . . . . . . . . . 32 
3.1 .1 CIE Guidelines for Coordinated Research . . . . . . . . . . 33

3.1 .2 Established Experimental Methods . . . . . . . . . . . . . 35

3.2 The Proof of Concept . . . . . . . . . . . . . . . . . . . . . . . . . 39

$3.2 .1 \quad$ Experiment Specifics . . . . . . . . . . . . . . . . 41

3.2 .2 Paired Comparisons . . . . . . . . . . . . . . . 44

$3.2 .3 \quad$ Following the CIE Guidelines . . . . . . . . . . . . . . . . 46

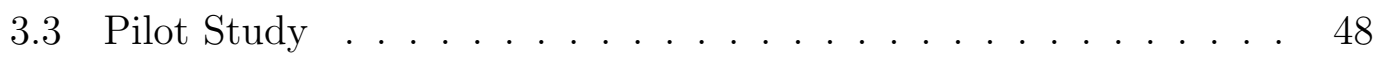

3.3.1 App details . . . . . . . . . . . . . . . . . . 49

3.3 .2 The Colors Chosen . . . . . . . . . . . . . . . . . . 50

3.3 .3 Statistical Verification . . . . . . . . . . . . . . 50

3.4 Future Work . . . . . . . . . . . . . . . . . . . . . . . 53

3.4 .1 Crowdsourcing . . . . . . . . . . . . . . . . . 53

3.4 .2 Mobile as a Platform . . . . . . . . . . . . . . 56

\begin{tabular}{|lll}
\hline 4 & Conclusion & 61
\end{tabular}

\begin{tabular}{ll}
\hline Bibliography & 64 \\
\hline
\end{tabular} 


\section{LIST OF TABLES}

$3.1 \quad$ Colors used in pilot study . . . . . . . . . . . . . . . . . 51

3.2 Expected/Average Rank, and Standard Deviation/Error of Ranks per Color (row totals) $\ldots \ldots \ldots \ldots \ldots \ldots \ldots$ 


\section{LIST OF FIGURES}

$1.1 \quad$ CIE 1931 xy Color Space Diagram [7] . . . . . . . . . . . . . . . . 3

1.2 How Humans Perceive Colors [25] . . . . . . . . . . . . . . . . . 4

\begin{tabular}{llll}
\hline 1.3 & sRGB gamut drawn inside the CIE 1931 chromaticity diagram [7] & 10
\end{tabular}

$2.1 \quad$ An example of the Helmholtz-Kohlrausch Effect where increased chroma appears to affect luminance . . . . . . . . . . . . . . 14

$2.2 \quad$ An example of chromatic crispening. The center colors are the same, while the surrounds chroma values differ. $\ldots \ldots . . . .15$

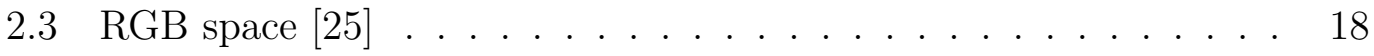

2.4 Spectral Power Distribution of the D65 Illuminant (modeled after

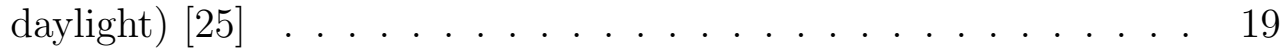

2.5 Cross section of the original device used during the Wright and Guild experiments [41] $\ldots \ldots \ldots \ldots \ldots$

2.6 The XYZ color matching functions that resulted from the Wright and Guild experiments [7] $\ldots \ldots \ldots \ldots \ldots . \ldots \ldots$

2.7 The three matching functions, each represented on their own axes, connected, and then projected onto an equilateral triangular plane

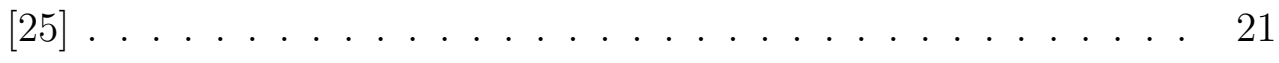

2.8 The 1931 CIE Chromaticity Diagram [25] . . . . . . . . . . . 22

2.9 Ellipses from MacAdam's solitary participant, Perley G. Nutting Jr. plotted on the CIE 1931 chromaticity diagram (ellipses ten times actual size). [7] . . . . . . . . . . . . . . . . . . 24

2.10 The Munsell Color Order System [7] . . . . . . . . . . . . . . 27

$3.1 \quad$ A screenshot of the proof-of-concept app . . . . . . . . . . . . 41 
3.2 An example of generating the paired comparisons tests for a test session using 3 comparison colors (reds) and 1 reference color (purple) 43

3.3 Pointer's gamut compared with the iPhone 5's [6] . . . . . . . . . 58 


\section{CHAPTER 1}

\section{Introduction}

Color is neither an objective nor absolute attribute of anything in our world; it is always a personal and subjective experience. While color experiences are generally coupled to real-world stimuli, it is important to remember that they can be affected by other stimuli in context such as motion, form, ambient light, and can even arise without stimulus entirely through dreams and memory recollection [20, p. 52]. Ultimately color is just one end result of the body and brain processing visual stimuli. All this makes quantifying the psychophysical phenomenon of color challenging but not impossible.

Aristotle and Newton were among the first to try to classify, understand, and order color. Many others have come up various other ways of classifying and ordering color, either by rough means of categories such as "reds" and "orange reds," by more qualitative means by which colors are divided by chroma, saturation, or lightness, and finally in more quantified scales using the same kinds of divisions mentioned prior. The holy grail of color science is a model of color which allows continuous (as opposed to discrete) measurement of colors and is perceptually uniform - that is to say if the model is represented in space, chang- 
ing a color within that space through any of its dimensions will have its changes uniformly reflected by human perception. At the moment, creating a perceptually uniform space that attempts to mirror human perception, requires ample empirical data.

Currently the most perceptually uniform space in the color science field is the standarized International Commission on Illumination (CIE) Lab 1976 space — derived from the CIE 1931 space which was built from data collected in 1920. The current standard for measuring distances between colors in this space is the CIEDE 2000 formula. While the newest Lab space and distance formulas are still largely based on data collected nearly a century ago, they have been modified using more recent data to become what they are today. The CIEDE 2000 formula only predicts the set of perception data that it is based on with about $33 \%$ accuracy [18]. It could very well be that inter-observer variability is so high that even a color model that perfectly mimics the average-world observer is poor at predicting individual perception accurately, but it seems unlikely that the figure would be this high. Considering the dubious quality and small sample sizes of the data that so much of the field is based on, it seems like a good idea to evaluate methods of leveraging new technology to assist in creating a better model of average-world observer to better model human perception.

The tests run in 1920 by David Wright and John Guild led to the creation of the $2^{\circ}$ Standard Observer that ultimately backed the creation of the CIE 1931 color space [24]. Their testing procedure was a color matching experiment where 7 subjects [31, p. 14] viewed multiple pure spectral colors (like the light of a laser) through a hole that forced the subject to only use a $2^{\circ}$ section of the fovea at the center of the retina, which at the time was thought to be the location of the majority of cone cells (we know now cones are concentrated over a larger area 
and $10^{\circ}$ is a better limitation). Participants mixed red (650nm), green $(530 \mathrm{~nm})$, and blue $(460 \mathrm{~nm})$ lights until they decided that the mix acceptably matched the provided reference color.

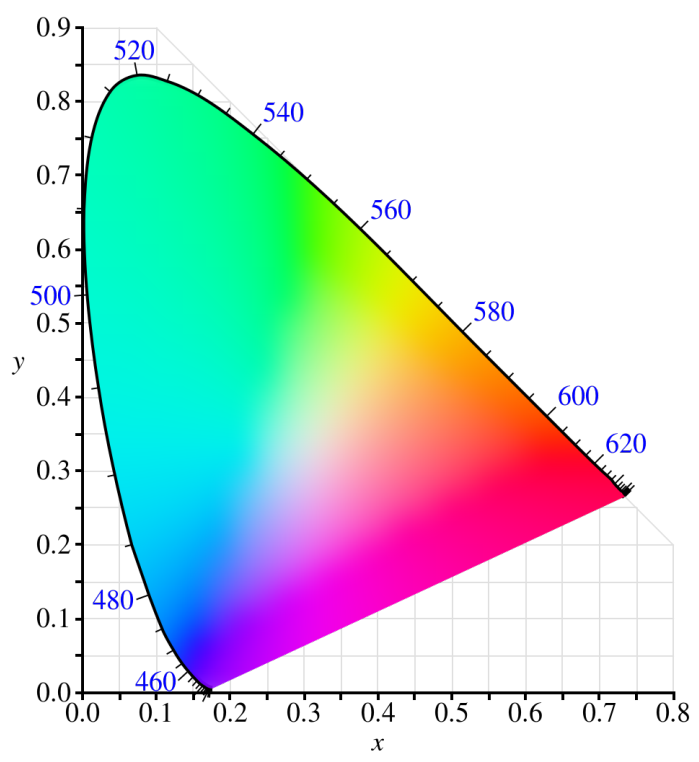

Figure 1.1: CIE 1931 xy Color Space Diagram [7]
The recordings from this experiment led to the creation of the CIE Standard Observer functions (Figure 2.5), which show us the mixtures of light required by a color-normal person's three cones to create a particular color experience. Through a series of transformations this diagram can be transcribed into the CIE $1931 \mathrm{XYZ}$ chromaticity diagram [17].

In 1964 after decades of criticism, the 1931 standard was re-

tired and replaced. Some of the criticism arose from other testing that seemed to challenge the testing the standard was based on, or highlight the lack of perceptual uniformity in the derived Lab space [17, p. 364]. A large part of this criticism came from the results of MacAdam, who using one subject, mapped ellipses on the CIE $2^{\circ}$ chromaticity diagram, the boundaries of which encapsulated all colors which the observer could not tell the difference from the color at the center of the ellipse [20, p. 142]. Tests like MacAdam's, despite using one observer, helped advance the field. His results were used as a guide to "warp" existing spaces into more perceptually uniform ones, and are still heavily referenced today.

Although a wealth of the research in the field of color science is from the 
mid 20th century, it should not be discredited. The efforts made then sparked the mathematical study of the field and were valiant ones given technology and knowledge available at the time. This said, experimentation has not advanced significantly since then, while we have proven that those experimental methods even when carried out carefully, still can lead to large variability and error [18]. Knowing this gives hope that maybe a different approach that embraces the nature of noisy data, by gathering statistically significant amounts of it can help us draw better conclusions in the future.

\subsection{Problematic Perception}

In attempting to quantify how humans perceive color there are three layers to keep in mind: the hard to predict physical factors that affect the way an object reflects and gives off light, the varying physiology of humans, and the unique black box that is the brain.

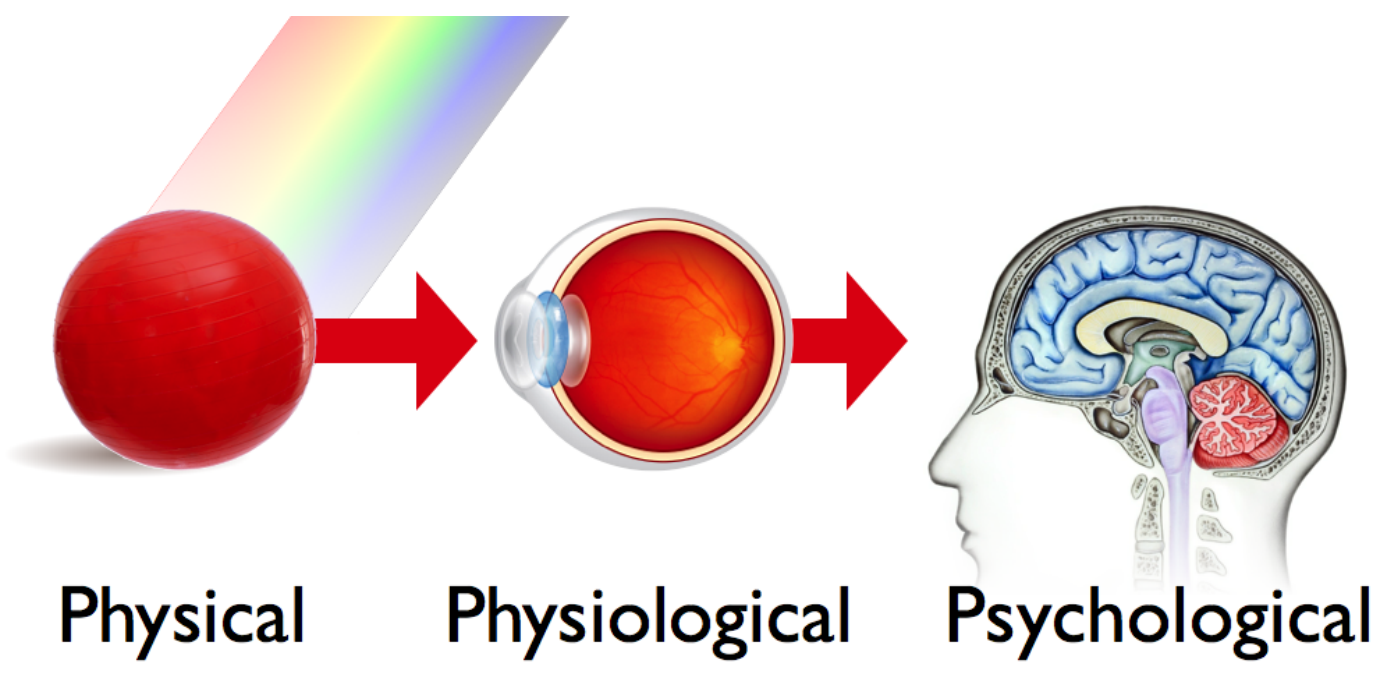

Figure 1.2: How Humans Perceive Colors [25] 
Considering these variables and challenges there have been what can be described as heroic attempts in the last century to understand and quantify the human perception of color. Improvements in modeling human perception can only truly come from empirical data [20, p. 31]. This is the field's largest source of error and headache. A large contributor to the color science field, Rolf Kuehni, sums up the need for better data in his 2008 paper, Color Distance Formulas: An Unsatisfactory State of Affairs, making a case that even the best current formulas only have at best a perceptual modeling accuracy of about $65 \%$ when tested on current color perception data sets [18]. Kuehni attributes this unsatisfactory measure at least partially to the fact that current formulas are based on "various sets of empirical difference perception data established with different kinds of materials, under different evaluation condition, and with different observer panels" [18]. The derived formulas are then often compared back to some of the very datasets that created them to be verified and mark the field's progress. There is no real alternative to using empirical datasets to create an "average observer" to derive these formulas, but through gathering data that is better than what we have we could potentially create an improved model of the "average observer" and in turn a better model of color difference.

\section{$1.2 \quad$ Limited Data}

Ultimately, colors are psychological experiences and while many are quick to point out that our understanding of neurological processes is improving, no matter what advances we make in spying on these internal processes, a proper understanding of the connection between what can be objectively measured and subjective experiences created in our minds (ie. consciousness) is far off. To this 
day "there is no detailed scientific explanation of the color vision process or the vision process in general" [20, p. 23].

Color scientists have noble goals in maintaining rigorous testing procedures in a field that relies on empirical data from the less than scientifically sacred or mathematically rigorous platform of consciousness, but ultimately well-controlled data still leaves us making conjectured leaps to create our models. This obviously makes the data gathered the crux of our current understanding. Restrictions in testing such as controlled light booths, expensive color-swatch sets, and other variable-limiting conditions (such as restricting subjects' fields of view to the 2 or 10 degrees where cone cells are densest in the fovea) have helped us obtain data with rigor, but have resulted in small and arguably somewhat homogeneous data sets.

Subjects can disagree significantly about the perceived magnitude of difference between two samples of color [13, 18]; so "for the data to have statistical useful meaning, they should represent the "world-average color difference observer" [20]. Based on existing data Kuehni thought this might require upwards of 60 colornormal observers [20]. Most of the existing experimental data used to the fit color difference formulas have used significantly less observers [20]. Trying to gather a relatively large amount of observational data using such rigorous restrictions has not yet proven practical or been incentivized enough for a contending model to be created. As Kuehni states, reaching such a goal will likely take a well-funded and orchestrated study [18]. Because of these reasons there currently aren't any significantly better datasets or new competitive color models. Therefore it may be worth considering new alternative methods of gathering data such as crowdsourcing.

If the reason for this unsatisfactory state of affairs truly is a lack of data as 
opposed to the quality of the data collected, it may be worth exploring crowd sourced data collection as a new experimental method. Even if this method proves not viable it could help us understand the importance of quality versus quantity of color perception data.

My thesis proposes new methods and tools for crowdsourcing color perception data collection via mobile devices rather than controlled light rooms with tiled samples in order to gather a massive, and potentially more comprehensive data set. This data could potentially be used in new, previously impractical ways to draw insights about how location of individuals, time of day, device profiles, etc affect perception. This method also makes it relatively easy to record additional experimental information such as how long observers spent on parts of the experiment and opens to door to ongoing testing with the same observer by pushed updates that allow for testing of new perception experiments when needed. After statistically accounting for noise and device shortcomings, the usefulness of the data gathered via this method is admittedly still a bit dubious, but there is great potential in a quantity over quality approach - the extent of which is currently unknown. I believe given a large enough dataset with the right statistical approach many of the envisioned shortcomings would ultimately have negligible effect on the accuracy of the conclusions drawn (this effect may be amplified on small or just-noticeable difference color difference data). Such a large and potentially global dataset could give us new insights into inter- and intra-observer variability, how environmental and testing variables affect perception, and more. So while it is unclear if a less groomed but potentially massive data-set of color perception data could help improve our current models of perception, exploring the idea is certainly a worthwhile endeavor. 


\subsection{Crowdsourcing Solution}

Given the ubiquity of mobile devices with their increasing color accuracy [26], it makes sense to try to leverage their pervasiveness to gather more voluminous, varied data to attempt to advance the field. Currently if someone wanted to create a new color model they would need to reinterpret preexisting data, buy or create color testing samples along with a controlled light viewing equipment, or build new experimental equipment. Crowdsourcing requires neither a grant-sized budget to build or buy new testing equipment nor physical access to a diverse set of willing test participants. This thesis aims to cover the foundations required to understand color and human perception and to investigate and provide possible tools and future directions we can take to bring color data collection and the color models and formulas that rely on them to new heights of perceptual accuracy.

As outlined, while promising, crowd-sourcing as a means of data-collection is problematic. Digital screens found on mobile devices use device-dependent RGB color spaces and represent a fraction of the colors humans can perceive [10, 20]. This means that even if we understand the perception of this limited subset of colors perfectly we would not be able to safely extrapolate that understanding to all of the colors humans perceive. Even if we can build better RGB displays with larger gamuts, creating all human visible colors by mixing three base colors is fundamentally impossible. While new technology like quantum dot displays are just beginning to enter the consumer realm they would likely only improve representation of the visual spectrum from the $20 \%$ seen in typical LCDs to $60 \%$ [10].

Until very recently most mobile devices had poor color management even if they had decent color reproduction. Essentially without color management there 
is no guarantee that a color displayed on one device will appear the same on another. Newer devices like Apple's iPhone 5 are color managed and use the device independent sRGB gamut meaning color can more strictly be controlled and predicted [16, 26]. Despite this revelation, even sRGB still only represents a subset of visible colors (see Figure 1.3); that said it still comprises a substantial amount of the object colors we see day-to-day [5] and if we can make progress modeling perception of the RGB gamut more accurately, that progress would likely spill into the rest of the visible range to some degree.

The problems reproducing all colors aside, knowing what color a particular viewer is seeing on mobile devices can become further complicated when we consider the effects of surrounding light, screen brightness, viewing angle, and the fact that screen pixels produce light rather than just reflect it they way real world objects do. Testing on a mobile phone will never be the same as a controlled light booth, but there are some rudimentary ways in which we can mitigate these concerns to a degree. The first of which is to group data by device; by doing this we can better guarantee a group is seeing the same colors. By controlling screen brightness we can make sure all observers are using the same brightness settings. By recording ambient light via device sensor or front facing camera we can roughly estimate ambient light (although we cannot capture the spectral distribution) to either again group data, throw out outliers, normalize variance, or just study the affects of surrounding light. By recording gyroscope and accelerometer data we can potentially determine device angle to assist the user in holding the device at the right angle, or know if they have moved the device significantly during the test. By recording all of this additional data I believe we should be able to filter or adjust the collected data enough such that the noise is not statistically significant. 


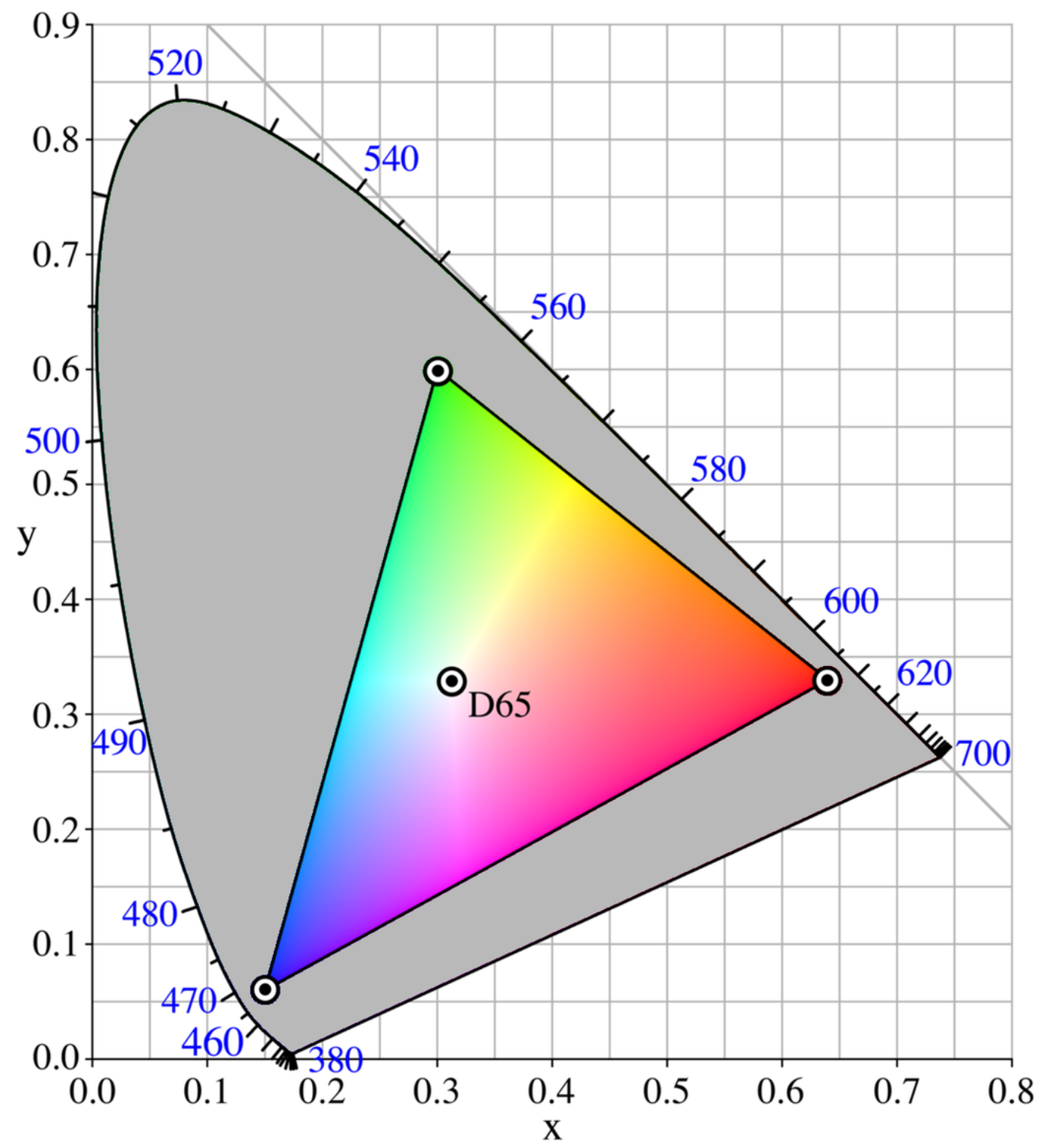

Figure 1.3: sRGB gamut drawn inside the CIE 1931 chromaticity diagram [7] 
While there is no logically sound way to verify a tool for measuring perception, we can try to use the tool to reproduce existing results from other experiments as a rough means of affirmation. In the case that the tool provides better, but different data than existing tests it will be hard to confirm that it is valid. That said, considering all of the variables mentioned it is unlikely this tool will collect more accurate data on a test by test basis, but by analyzing the overall results we should hope to see similar results to other tests.

While I cannot claim to be color scientist let alone a mathematician or statistician, I've studied and tried to understand the field to the best of my abilities so that I can apply my expertise as a computer scientist to help advance the color science field by way of new color discernment and discrimination testing tools built to tap the masses. 


\section{CHAPTER 2}

\section{Background}

\subsection{Color}

Color is arguably the most important sense humans have for navigating, understanding, and internalizing the world around us. Color itself is a vague term; a more clarifying way to define color is as a color experience, however, for simplicity's sake we will just refer to this as color. At its core, the experience of vision is formed by a small range of electromagnetic waves that are detected and interpreted by the roughly 120 million photosensitive cone and rod cells in our retinas. Only 7 million of those are cone cells that help us detect color, the majority of which are packed into a $0.3 \mathrm{~mm}$ across circular area ${ }^{1}$ called the fovea [20, 3]. From here a complex patterns of nerves interpolates this information in ways largely unknown before further processing and interpretation in the brain [20, p. 39]. Most of us are trichromatic and have three kinds of cone cells. A non-trivial part of the male, global population is dichromatic, causing partial color blindness.

\footnotetext{
${ }^{1}$ Tests that refer to a $2^{\circ}$ observer restrict observer viewing to this dense, cone-only area of vision.
} 
There's evidence that an unknown, small portion of the female population may be tetrachromatic having slightly extended color vision [17, 15]). These three kinds of cone cells are most sensitive to what we refer to as long, medium, and short wavelengths, which roughly associate with the colors red, green, and blue respectively. Purely talking about the signals our brain receives these are the only real color inputs we receive, but once in the brain these signals are combined to form new color experiences like yellow (from reds and greens) and magenta (from reds and blues). This is to say that even when we produce a pure wavelength of

yellow light, the color we perceive is actually being formed from stimulation of the long and medium wavelength cones. The spectrum of color we can perceive is generally referred to as hue, and while it is only one part of what makes up a color, as we'll see later is regarded as one of the most important aspects when humans differentiate between colors [17, 20].

\subsection{Modeling and Ordering Color}

There are many colorspaces in which we can quantify and numerically represent colors. Different colorspaces often define new component systems for breaking down colors, but often just warp and transform the space to better fit a set of data or situation at hand. Most people tend to think of color in three components: hue (what we tend to think of as defining color), saturation (the strength of the color from grey to pure), and lightness (how light or dark a color is). There are other component systems where the space's dimensions are based on concepts like opponent colors, or even on the abstract tristimulus responses of the cones. Still, all of our current models are likely over simplifications of how colors are actually created and held in the human mind. There is no sound reasoning aside 
from familiarity and convenience that most color spaces are Euclidian in nature? In order to improve our simple models over the years we have warped the spaces into contorted shapes that attempt to better represent perception, and when that hasn't worked we've modified the way we measure distances in that space via distances formulas, which we will discuss in detail later.

Because of the nature of the perception, it is challenging to pare the problem down into simple numbers and to understand the perception of colors without taking into account their context, but we can still come close. Effects like metameric matching (objects that appear to be the same/different colors under different light sources), the Helmholtz-Kohlrausch effect (see Figure 2.1), and chromatic crispening (see figure 2.2 can alter human perception of color. On top of these psychological effects, genetics, biological variance, hormones, and age related corneal yellowing [30] further affect our vision. For the sake of this discussion and research we will largely, and somewhat naively, ignore these complications (color vision deficiencies excluded), although they are ultimately a part of developing a truly perfect, perceptually uniform color space.
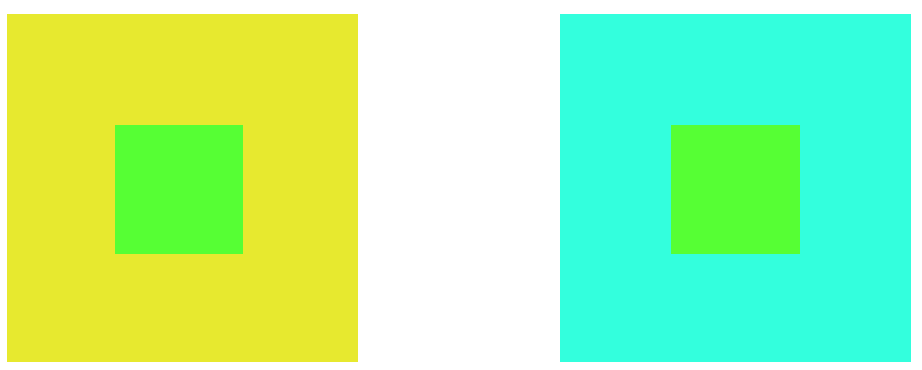

Figure 2.1: An example of the Helmholtz-Kohlrausch Effect where increased chroma appears to affect luminance

\footnotetext{
${ }^{2}$ Some data supports that this is a strange state of affairs and a recent study has even proposed a new color space that relies on six dimensions with colors as manifolds of a complex hypersphere [28].
} 

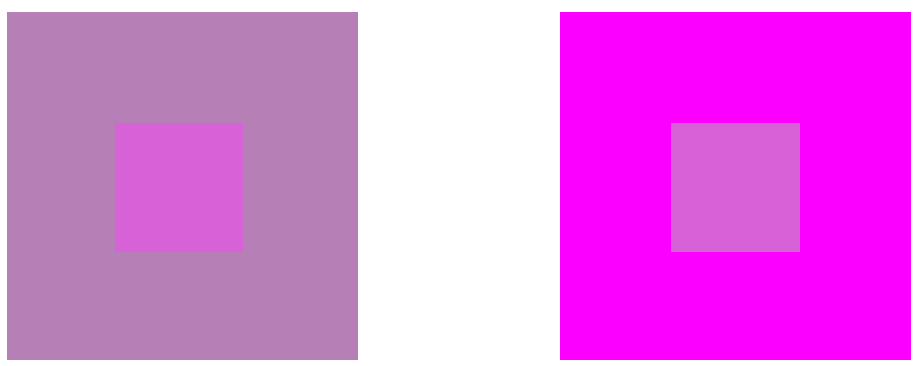

Figure 2.2: An example of chromatic crispening. The center colors are the same, while the surrounds chroma values differ.

\subsection{Perceptual Uniformity}

Quantification of color with accurate attention to human perception has been an ongoing struggle first documented by Ancient Greeks; it remains unsolved today and some say is impossible to solve [17]. Put simply, forming a perceptually uniform space means designing a color space with humans in mind before machines, one in which a measured distance between two colors points is proportional to their perceived distance. That is to say around each color is an imaginary sphere of arbitrary size at which all colors on its surface appear equally different from the center color, and if the radius were to be doubled all of the colors on the new sphere's surface would appear twice as different from the same center color. As mentioned there are many external and internal variables which complicate our understanding of perception by way of being largely private and subjective, and thereby unobservable and unverifiable. It can be argued that without solidifying our understanding of these pillars of perceptions we cannot make any scientifically sound headway in our understanding of human perception of color, which while true, as Kuehni says "appears simply to address the fact that at this point in time we do not have an understanding of consciousness" 20]. This is where the field of psychophysics (the study of the psychological connection between physical stimuli and mental experiences) draws scientific criticism. In the 
absence of an analytically rigorous foundation we must do our best to empirically draw and rigorously test the observable and coincidental relationships of human perception.

Since perceptual uniformity is not something can be logically solved even if we could measure the physiological responses of our cones (including the intermediate cells between the cones and the brain whose purpose we still don't understand [20]), more accurately we would still be lacking clarity on the final black box step of the brain where the final color experience is formed. Therefore all color spaces that aim to achieve greater perceptual uniformity must be based on experimental datd 3 . While standards bodies such as the Commission Internationale de l'Éclairage $\unlhd^{4}$ (CIE), Optical Society of America (OSA), and others have described standard spaces and data sets, the field is divided when it comes to following standards. In the past, standards have been created out of necessity of a divided field, or community discoveries and new experimentation.

\subsection{Established color spaces}

Color spaces provide an abstract mathematical way to model and understand colors and enable quantification of colors as coordinate tuples using axes that are appropriate for the task at hand. For instance while RGB provides a suitable model for manipulating the colors of display hardware by representing pixels, CMYK is a more fitting model for print work by representing ink. We can move colors between spaces but there can be a loss of accuracy in the process through

\footnotetext{
${ }^{3}$ It's important to note that many spaces are naively labeled as perceptually uniform when they really are just striving for perceptually uniformity and may only come relatively close to it.

${ }^{4}$ Translates in English to International Commission on Illumination.
} 
gamut clipping, which occurs when a source color space can model more colors than the target one. Often moving from one space to another requires additional information especially when moving from abstract spaces to the more tangible ones where say how lights interact with and change colors is important.

There seemingly isn't a particularly sound reason that color should be modeled using a 3D Euclidean form but whether out of the convenience of its familiarity or because most of us have three types of cones, they typically are. 3D color spaces can generally be divided into two camps: those sized by the abstract principles of a task (eg. RGB, CMYK, or HSV), and the rest which all have scales that attempt to balance each other to for a gestalt of perceptual uniformity. Spaces that attempt perceptual uniformity often feature new axes and odd contortions so that a Euclidean distance between two color points is proportional to human perception of color difference. A helpful way to understand the concept of perceptual uniformity is to look at a space where it is poor such as RGB; Looking at figure 2.3, if we measure the 3D Euclidean distance between $\mathrm{A}$ and $\mathrm{B}$ along the RGB axes their color difference is $\mathrm{X}$, the same is true for $\mathrm{C}$ and $\mathrm{D}$, but anyone with normal vision will disagree that the pairs are equally different.

\subsubsection{RGB}

The RGB color space is based more on display technology than human perception. In RGB colors are defined by their red, green, blue components. The space is represented by a cube of equal length axes with white at the combined maximums and black at the combined minimums of the origin. The entire gamut of possible color (in RGB space) fits neatly into a 3D Cartesian cube, but dramatically fails at being perceptually uniform. This is easily evidenced by looking 


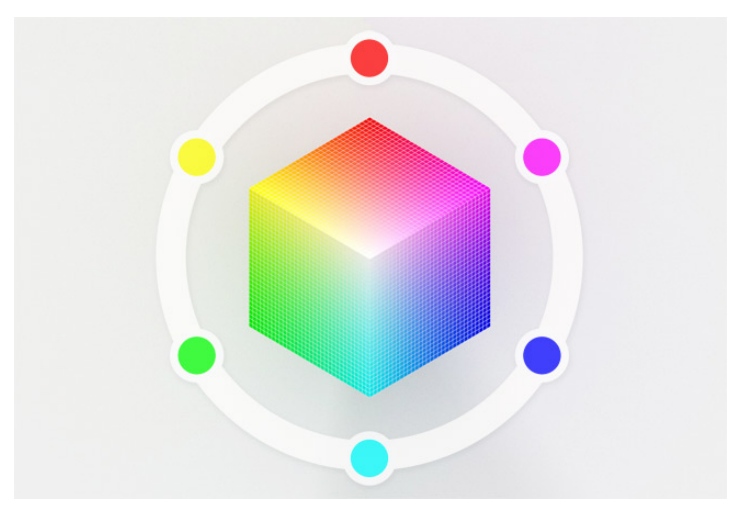

Figure 2.3: RGB space [25]

at the corners of the space where moving equally down any of the three edges results in colors that any normal-vision person would quickly notice are no where near equally different.

\subsubsection{HSV \& HSL}

HSV and HSL stand for hue, saturation, and value/lightness respectively, which makes both crudely attuned to our intuitive understanding of - and thereby perception - of color. These two spaces were developed in the mid 70s by geometrically mapping RGB into a cylindrical space [17]. Both map the RGB space similarly except when it comes to the value and lightness axes; where the HSV model has the white point as the "core" of the cylindrical space so changes in the value axis never change the "whiteness" of a color, the HSL space has lightness as a top plane causing the color to approach white, which is represented by the entire top surface of the space rather than as a core. 


\subsubsection{CIE XYZ}

The International Commission on Illumination defined the CIE XYZ space in 1931 by way of a set of phenomenal efforts given technology at the time. To understand the XYZ space it is key to remember that color is not an absolute property of an object and is merely a perception of reflected and emitted light interpreted by our cones and the brain. Like white light through a prism it is possible to split colored light (like that reflected from an object) into its discrete measurable color components. It should be noted that this is not possible with pure spectral light-sources like a laser.

Spectral power distributions (SPD) are an objective way to measure color if perception is not a consideration - this is color at its purest in a mathematical sense. Seen in figure 2.4 is the SPD of the D65 standard daylight illuminant

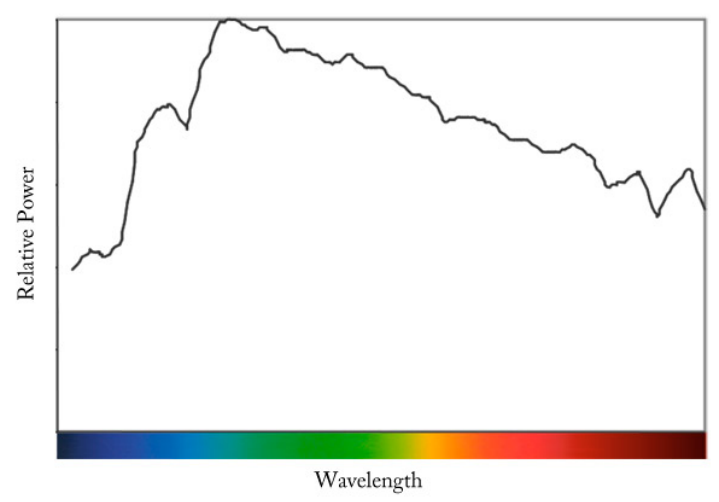
showing the colors that make up a typical "white" daylight. Because of the nature of our cones, a SPD Figure 2.4: Spectral Power Distribution of the D65 Illuminant (modeled after daylight) [25]

of the light that peaks in the green wavelength and an SPD that spikes in the yellows and blues could represent the same real world green; both of these SPDs would essentially elicit the same cone responses that form the sensation of green. Knowing this concept helps understand the experiments carried out by William David Wright and John Guild in the 1920s. Using a complex contraption they generated pure spectral colors and had ten normal-vision observers adjust lights 
corresponding to the primary colors of red $(650 \mathrm{~nm})$, green $(530 \mathrm{~nm})$, and blue (460nm) until they were satisfied that their mixed color matched the pure one. Values varied between observers, the consequence of which is up for debate, but besides varying genetics, could also include to age related yellowing of the macula in the foveal region of the eye [11].

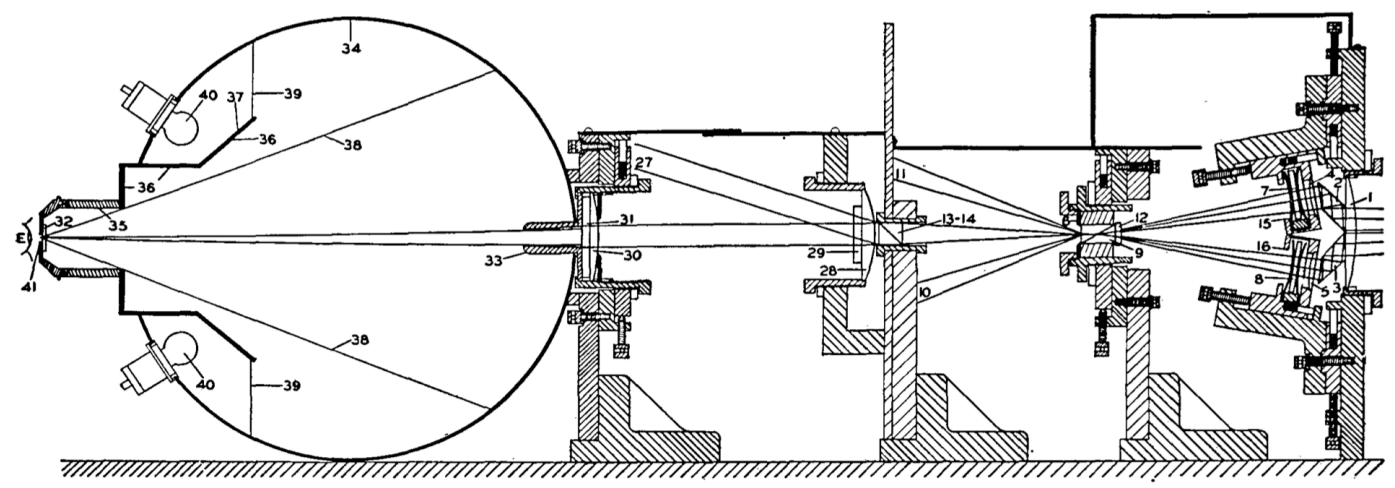

FIG. 2. Vertical cross section of chromaticity discrimination apparatus.

Figure 2.5: Cross section of the original device used during the Wright and Guild experiments [41]

With this data, they were able to create the XYZ color matching functions to represent what mixes of the primaries which directly affect the cones are needed to create the color experience as that of a pure spectral color. This ultimately defines the CIE's Standard Observer which is the basis of most color research. This standard observer in essence, is a table of values that backs these functions. 


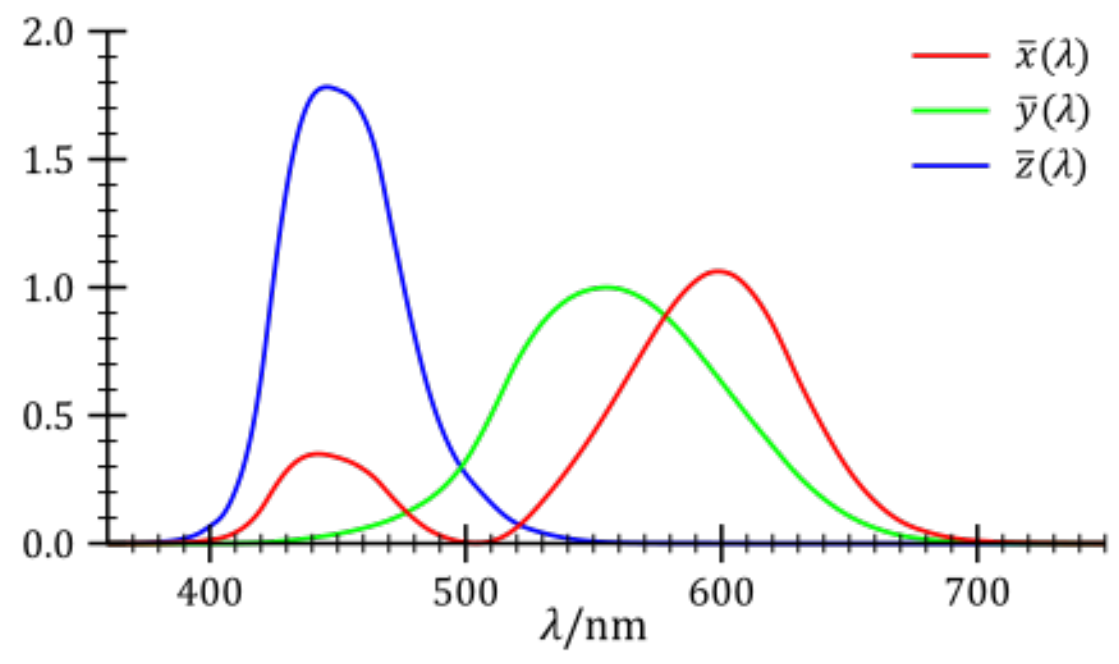

Figure 2.6: The XYZ color matching functions that resulted from the Wright and Guild experiments [7]

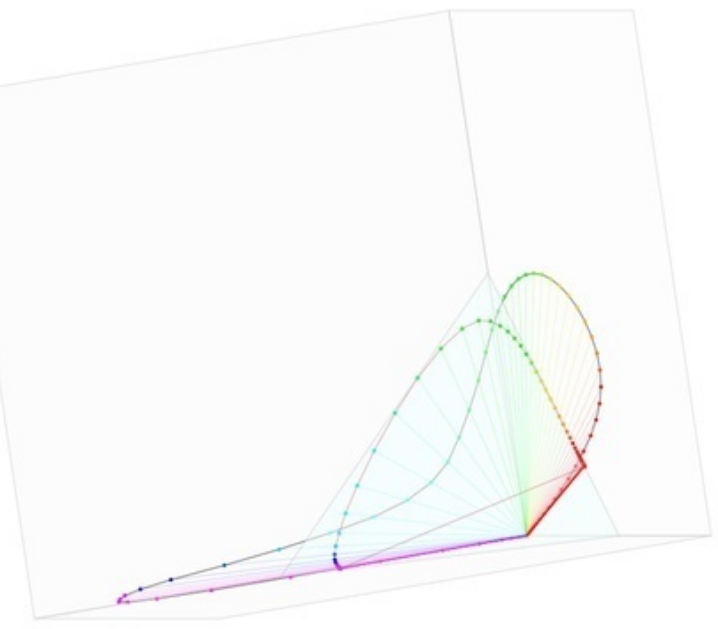

Figure 2.7: The three matching functions, each represented on their own axes, connected, and then projected onto an equilateral triangular plane [25]

By mapping each tristimulus value function in its own dimension of a three dimensional space we end up with something seen in the outer shape of figure 2.7. 
By then projecting these functions onto a triangular plane (Figure 2.7) formed by connecting the equal maximums of each axes we end up with a familiar standard: the CIE 1931 Chromaticity Diagram (Figure 2.8). The Chromaticity Diagram is an often referenced diagram, and while useful is an awkward diagram to reference since it leaves out the luminance dimension of color. The diagram's usefulness comes from the ability to take any two color points, draw a line connecting them, and know that the end points of that line can be combined to form any color along that line. Going further, if three points are chosen, any color within that triangle can be created by mixing those end-points. This is the basis of RGB displays or CMYK printing. An important aside is that the color of the diagram cannot be accurately represented on a screen or paper and thus many of the colors that should be shown in Figure 2.8 are impossible to represent in this form.

It should be noted that the Standard Observer referred to previously is specifically the CIE $2^{\circ}$ Standard Observer, which gets its name from the fact that during the experiment the subjects field of view was constricted to $2^{\circ}$ based on the erroneous assumption that all of the cones in the eye were located in a $2^{\circ}$ section of the fovea in the back of the eye;

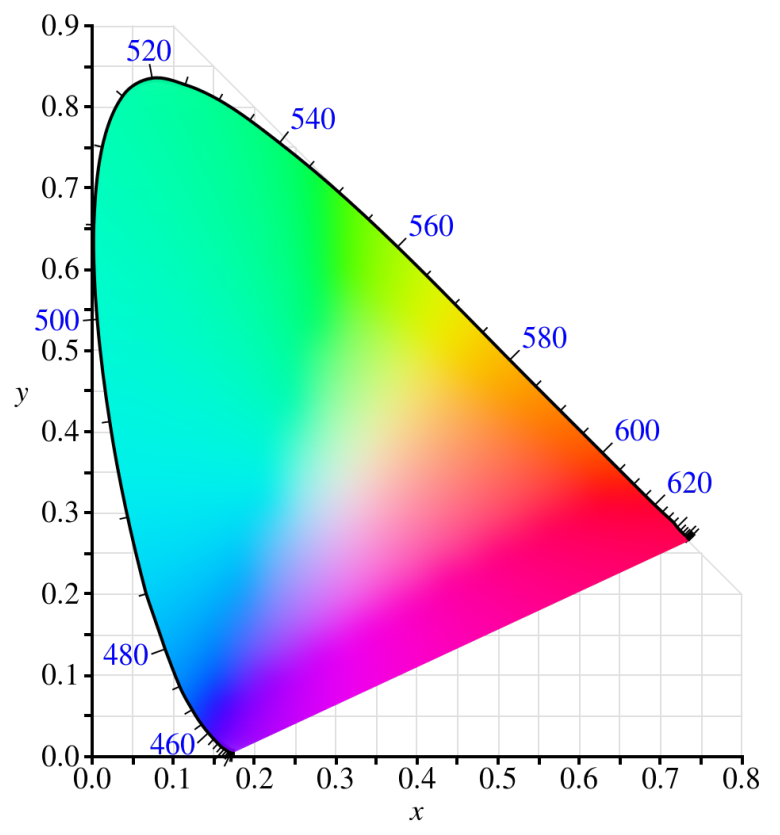

Figure 2.8: The 1931 CIE Chromaticity Diagram [25]

while this is where the largest 
concentration of cones is, quite a few lie outside this range leading to the creation of the $10^{\circ}$ Standard Observer in 1964 [17] which as expected is slightly different and recommended by the CIE as most appropriate for representing the response of human observers.

In summary the XYZ space is a mathematically defined color spaces that represents all color experiences a normal color observer can have. It is often used as a base from which other color spaces are created and can serve as a useful intermediary when translating between color spaces.

\subsubsection{CIE Lab}

The CIE Lab space (technically L*a*b*) was developed by the CIE in the 70s out of a need to unify many disparate color spaces and distance algorithms that were quickly popping up in industries dependent on color. The $L$ component of the space represents luminosity and ranges from 0-100, while the $a$ and $b$ components represents red-green and blue-yellow ranging from -128 to +128 ; These two color pairs are opponent colors that don't generally exist at the same time [17].

After MacAdam first plotted his ellipses (representing sets of colors that were within just noticeable difference from reference colors) on the 1931 CIE chromaticity diagram he set out to apply linear transforms to the space in such a way that ellipses would become equally-sized circles and thus the space perceptually uniform according to his data. Many others made similar attempts during the 50s and 60s to little success. Ultimately amongst increasing new visual color difference data sets, formulas that relied on MacAdam's ellipses were worse. In 1948 R. S. Hunter started making tristimulus based colorimeter devices that could de- 


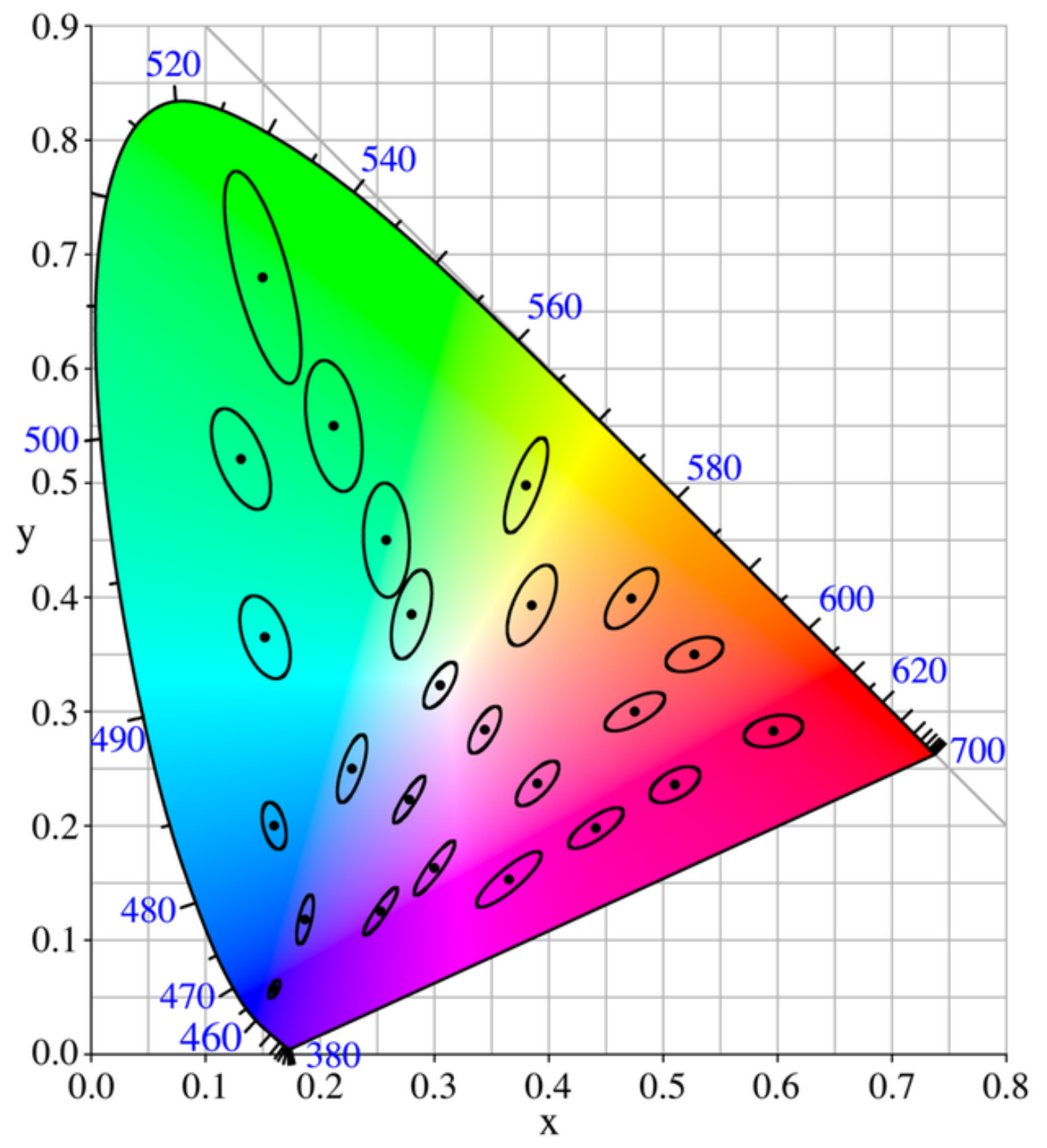

Figure 2.9: Ellipses from MacAdam's solitary participant, Perley G. Nutting Jr. plotted on the CIE 1931 chromaticity diagram (ellipses ten times actual size). [7] 
termine CIE tristimulus values of real life colors. Hunter proposed a new color model with three axes $(L, a$, and $b)$ as a translation of the XYZ space. The translation of the space was later revised to agree more closely with the Munsell color difference data (discussed later). By the 1960 there were at least a dozen or so datasets with related color models and new ways of measuring color differences, all created out of necessity by the industries relying on color measurement and reproduction (textiles, food, paint, printing, etc) [17]. In 1973 Kuehni found poor levels of correlation between all of these models and their data sets, and that they were generally poor at representing the average observer [17]. Various groups and individuals continued down the path of ellipsoid fitting; finally in 1975 R. M. Rich, F. W. Billmeyer, and W. G. Howe modeled ellipsoid fitting on a computer with great accuracy [17]. In 1976, out of necessity for an industry standard, the CIE established the Lab space we know today. It didn't make any advancements over existing functions and even drew debate about its quality compared to some of the existing functions it sought to replace, but ultimately a compromise was reached and it was deemed a standard [17].

The CIE Lab space has become the standard form of color measure it was intended to be. The space still draws criticism though, partially because the full range of the space actually exceeds human vision and contains colors that don't exist in human vision, but mainly because it still lacks strong perceptual uniformity. So while the space was formed with a goal of perceptual uniformity, it falls short. Lab space is fairly accurate in its perceptual modeling of luminosity, but is weakly perceptually uniform in the $a$ and $b$ axes, which affect the chromaticity. Humans are more sensitive to change in hue than any other color parameter (a phenomenon called hue superimportance) making improving perceptual uniformity in axes that adjust hue challenging. 
Since 1976, modifications, transformations, and translations of the space have been proposed. As we'll discuss, most of these changes have been rolled into modified distance formulas used within the space rather than changing the space itself. While there are other potentially better ways to model color, the CIE Lab standard has served well enough to provide common ground until such a time where a significantly better space can replace it.

\subsection{Color Atlases}

Where color spaces attempt to quantify colors on a continuous spectrum color atlases take a discrete approach, defining sets of specific, unique colors and mapping them onto planes of hue, brightness, etc. Color atlases were arguably one of the first practical ways color was ordered. There have been many color atlases, but we will discuss the two most important ones.

\subsubsection{Munsell}

The Munsell color order system was created by Professor Albert H. Munsell around 1905. It was the first 3D color order system that represented colors cylindrically in the three dimensions of hue, value, and chroma (similar to saturation) with an attempt at being perceptually uniform between steps in any dimension. In the 1930s the USDA adopted the Munsell color order system as a standard for quantifying the colors of soil samples [8]. The Munsell system was refined in

the 1950s and 1960s by the Committee on Uniform Color Scales of the Optical Society of America; through a series of perceptual experiments they completely revised the Munsell system and ultimately replaced it with the OSA-UCS color 


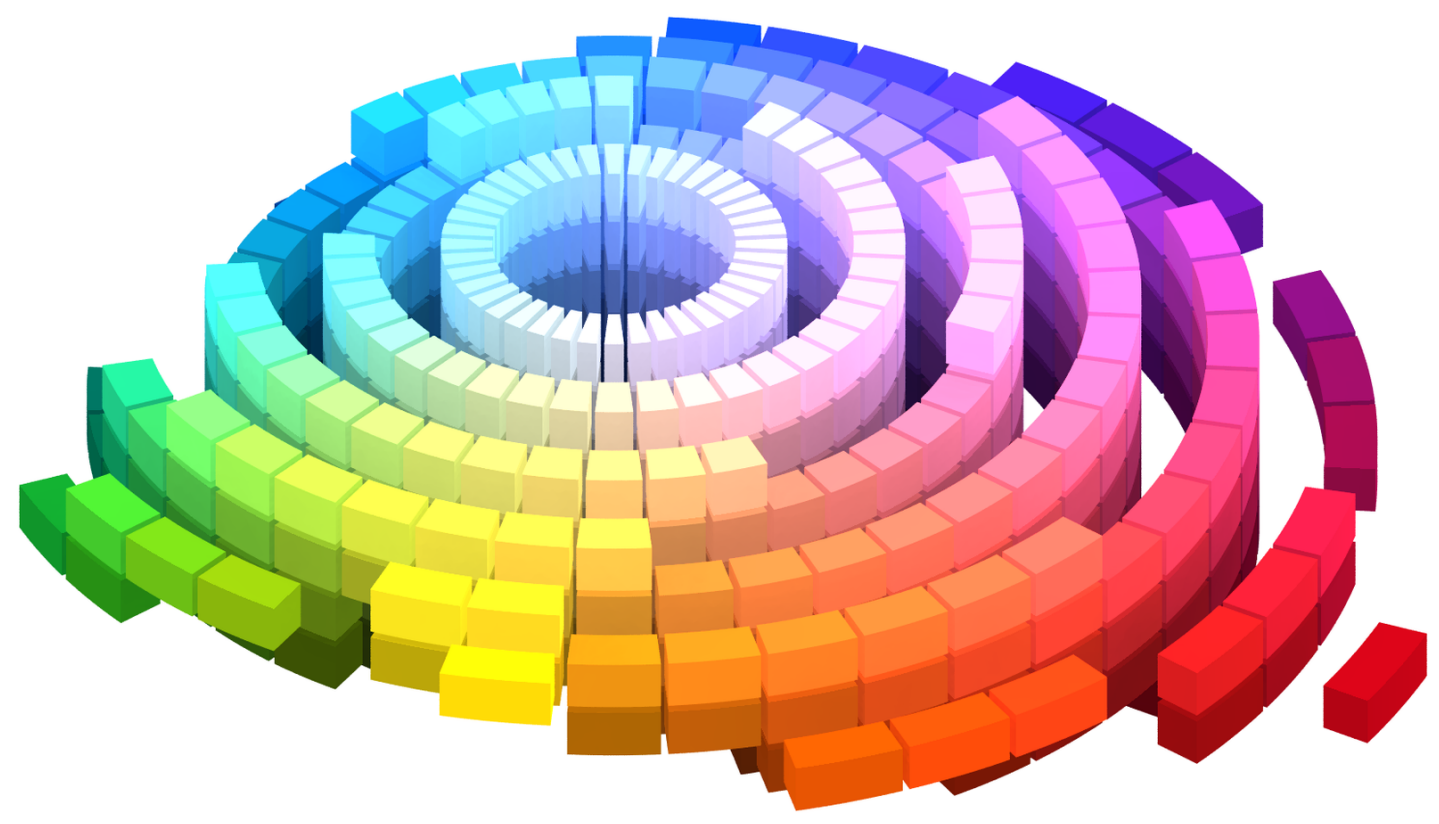

Figure 2.10: The Munsell Color Order System [7]

order system [17]. The Munsell system continues to have widespread use and is referred to and relied on in various industries from food quality control to car paint [8].

\subsubsection{Optical Society of America Uniform Color Scales (OSA-UCS)}

The Optical Society of America developed the Uniform Color Scales while attempting to improve the perceptual uniformity of the Munsell color order system. Through the development of new experimental data they found that modeling color with perceptual uniformity in a Euclidean space was not possible, but that they would come as close as possible. After studying 38 uniform color scaling studies a 500 color set was modeled in 3D rhombohedral lattice based on a cuboc- 
tahedron. Essentially OSA-UCS is a continuous enclosed space like any other; being based on the XYZ space using a $10^{\circ}$ standard observer and a D65 illuminant it is simply another way to discretely model color [17].

\subsection{Color Distance}

Quantifying the magnitude of difference between something that tastes salty and something that tastes sweet seems like a bit of a ridiculous task, yet this is not different from what color distance formulas attempt to do. Color distance formulas serve as numerical representations of how different two colors are, as perceived by humans, a problem that has been shown to be provably impossible to do with perfect accuracy [20]. As Kuehni puts it, "any model short of duplicating in a robotic fashion the complete color vision system will be found limited and inadequate in specific circumstances... and then there remains the issue of interobserver variability and its causes and imports" [20]. In the absence of being able to replicate the visual system, to at least quantify it relatively well we rely on empirical human experiments to build a model of the average world observer's color perception, with the purpose of shaping our mathematical models to fit that average observer's perception accordingly.

Many 3D color spaces have been built in an attempt to capture this average perceptual uniformity into their shape such that a Euclidean distance (the square root of the sum of each dimensional difference squared) is perceptually uniform no matter where the colors chosen lie in the space nor how different or similar they are. Models can only be as good as the experimental, subjective data they are based on though and whenever new datasets are gathered they tend to disagree with the existing data to a non-trivial degree [17]. Often this leads to new distance 
formulas that are far more complex than a simple Euclidean distance. These distance formulas are the crux of the field and forefront of research.

There are many different experimental color perception tests to test for different sized magnitudes of difference between colors, from large to just barely noticeable. On top of that, there are dozens of variables (from presentation of colors, to size, to illumination, to how questions are asked) that can affect the delicate subjectivity of the problem at hand [9]. Even when testing is done properly there is considerable error from inter-observer variation, and even intra-observer variation between testing sessions when conditions are maintained [13]. In the end even after creating a perfect world average observer, our mathematical predictions of the magnitude of difference a person will detect may only really be accurate for $20 \%$ of the population [20, p. 132]. These points, while discouraging also bring up a whole new facet of study around how different people perceive color, when their perception changes, and more, but before moving onto these goals we should first focus on improving our current best distance formulas to the best of our abilities while there is definite and measured room for improvement.

\subsubsection{The Curent State of The Art: CIEDE2000}

Near the end of the 19th century Hermann Helmholtz, one of the largest contributors to the visual perception and experimental psychology fields proposed measuring the difference between two colors using a Euclidean distance [20]. In the 1930's Dorothy Nickerson attempted to more accurately model perception of difference by introducing a psychological basis that took into account some of the strange effects of perception. Her work indicated that the well-respected Munsell system might not have had perceptually uniform axes [17]. In 1942 
David L. MacAdam published a new set of experimental data. In MacAdam's experiment one observer repeatedly mixed RGB primaries to match 25 colors (selected relatively evenly across the CIE chromaticity diagram). After gathering over 25,000 trials, MacAdam was able to derive the now iconic ellipses (see Figure 2.9) representing colors within a range of just noticeable difference (JND) 24]. Although these ellipses are still widely referred to today, when tested against other wider datasets that measure color perception of object colors they perform poorly [20]. The procedure of drawing ellipses to represent difference data remains today. A presumed reason that MacAdam's data might not match up to further studies is that color matching may happen at the physiological-level more than at the postprocessing level of hue and chroma evaluation in the mind. MacAdam's single observer data, while significant, cannot be taken to represent the average observer and what they perceive as just noticeably different. Even MacAdam recognized this in his 1942 publication: "some variations in judgment are inevitable. No doubt the ideal method would be to make a large number of matches at each point in the colour chart and then to analyse the spread of the observations, but in practice this would be an impossibly lengthy process" [24].

Following MacAdam's work many new studies emerged testing color difference perception of object colors to create ellipses 5 around reference colors. Fitting color ellipsoids to be spherical using mathematical transforms has become the standard process by which distance algorithms are created and improved [17]. Around the mid and late 1900s a significant amount of new data was gathered. With each new set of experimental data came new difference formulas, and "in 1973 Kuehni found that 13 different formulas were in use in color-related industries", but that "levels

\footnotetext{
${ }^{5}$ These ellipses almost always are more elliptical than circular and tend to "point" toward the somewhat-central neutral white point because of the heavy significance hue plays in perceived color difference.
} 
of correlation between visual and calculated data for all... were unsatisfactory" [17]. Eventually in the 1970s, out of need more than revelation, the CIE proposed a new standard: the CIELAB space and its respective Euclidean measure of difference.

Following a rash of new experimental datasets and distance formulas, the most recent crown jewel of the CIE is CIEDE2000, - the result of mathematically fitting of three combined ellipsoidal datasets (RIT-DuPont, Witt, Leeds and BFD-P) as well as six other considerations of perceptual effects that the CIE tried to account for ${ }^{6}$ that weren't always accounted for in previous formulas [13, 17, 20]. Despite best efforts, the exceedingly complex CIEDE2000 formula has shown to be anything but a large improvement over prior formulas and is still not anywhere close to perfect.

\footnotetext{
${ }^{6}$ The six perceptual effects are hue super importance, relative change in chroma difference with respect to chroma location, lightness crispening based on surround lightness, the fact that the ellipse of the white-point is still an ellipse, hue difference with respect to hue location, and ellipse rotations in the negative $\mathrm{b}$ axis.
} 


\section{CHAPTER 3}

\section{A New Way to Test}

\subsection{We Require Additional Data}

Often color science research papers concede to some degree in conclusion that while best efforts were made, due to the noise of variability, a larger, more diverse dataset might have made for stronger results [13, 19, 21, 22]. Additionally gathered datasets are often hard to compare to one another since they are all collected slightly differently. This has slowed field-wide progress and not greatly contributed to our knowledge about the degree to which these differing environmental conditions affect results. In addition to all this we're beginning to see evidence that observer variability might be something that cannot simply averaged out, and instead will require us to come up with many different color models tuned to different groups of the population [19].

All of this is unclear from the relatively small amount of data typically gathered [18, 13]. As mentioned earlier, the study of psychophysics stands on the unsound ground of consciousness, so rigorous testing methods are necessary for 
both respect and to not add additional variables to observing the complex device of the mind (and body to a degree). While carefully manufactured paint/fabric samples and calibrated light booths are invaluable assets in studying color, they are exceedingly expensive and require finding and bringing in test subjects one-by-one for lengthy experiments. Even experiments that use digital displays typically require custom setups and relatively large budgets or sponsorships for the results gathered [39]. The field bemoans more data, yet gathering it is often prohibitive and archaic.

\subsubsection{CIE Guidelines for Coordinated Research}

In the Fall of 1978, the CIE published a paper titled CIE Guidelines for Coordinated Research on Colour-Difference Evaluation with the goal of unifying research in the field shortly after the standardization of CIE Lab 1976. It was understood that the CIE 1976 standard was a stopgap that would ultimately need to be refined [17]. The task at hand was to establish a comprehensive set of color perception data focusing on small, medium, and large color differences under different viewing conditions. The authors admitted that the task was complex and may never be completed, but thought substantial progress could still be made with a strong collective effort. The established guidelines suggest breaking testing into four steps, with an end goal of forming contours of equal perceived colour difference $(\Delta E)$ from a particular color via a provided difference equation. The guidelines of the steps are as follows. 


\section{Step 1: Study of Methodology}

Step one dictates that experiments should be conducted in the neighborhood of five standardized color centers (roughly a grey, red, yellow, green, and blue) the last four of which are means of the four tetrahedra studied in the OSA Uniform Color Scales [9]. Observers should be tested to ensure that they are normal trichromats and color discrimination should be further tested if possible. The guidelines recommend repeating experiments 4 times with at least 20 observers.

The guidelines outline three different techniques of tests, summarized as colorimeters (light mixing devices), color difference simulators, and object colors (sample swatches). In regards to testing finite object colors, the CIE estimated that it would take about 50 samples per color center, well distributed along standard vectors from a color center. Essentially all of these tests involve comparing colors to a reference color in one way or another.

A couple of methods are suggested for comparing object colors, the first being the Rich-Billmeyer-Howe method where pairs of samples are judged for either matching or not matching; repeatedly testing the pairs helps establish if color pairs are consistently perceivable as different. A second method of comparison with a constant color difference provides observers with two pairs of colors, one being greyscale. Observers are asked to judge whether the difference between the greyscale pair is greater than or less than that of the standard pair. Again, this test repeats the testing of pairs to test for consistency. The third suggested method of comparison is the visual scaling method which is a catchall term for a group of experimental methods often used in psychology and sociology studies, but also psychophysical studies, where the end result is a ranking of tested items. Paired comparisons (discussed later) is a comparative visual 
scaling method, with associated statistical procedures that have been developed specifically for psychophysical tests.

\section{Step 2: Study of Experimental Parameters}

Step two suggests investigating the effects of the following parameters on perceived difference: sample size, illumination level (luminance level), sample separation, texture, colour surround (focusing on neutral surrounds and surrounds that are similar in color to the samples), luminance factor, size of $\Delta E$ of samples, observer variability, duration of observation, and monocular versus binocular observing.

\section{Step 3: Study of Color-Difference Perception of the Whole Color Space}

Step three is to derive a set of fitting coefficients constants throughout the color space by following a decided set of conditions (described in Step 2) using the data from the conducted experiment.

\section{Step 4: Derivation of a Distance Formula}

Step four is to derive a new color distance formula from the data obtained in Step 3 and to test it in field trials.

\subsubsection{Established Experimental Methods}

The CIE guidelines for coordinated research recommend one of three kinds of previously mentioned testing procedures. Through investigating dozens of color perception experiments conducted over the last century with a focus on the last 
few decades, we found a myriad of experimental set ups, but all more or less largely fall into the following four categories described in detail below.

\section{Color Mix Matching}

Color mix matching tests (like those used by MacAdam in the early 1900s) generally rely on mixing primary colors to match a reference color, with the end result being a set of colors that the observer has mixed and decided were indistinguishable from the reference. While this test could be implemented on a mobile device, its value is questionable for a couple of reasons. First, the spectral pure wavelength lights meant to represent wavelengths of peak recognition by the cones, lie on the edges of the chromaticity diagram and cannot be recreated by any RGB display. We could forgo this requirement and use the RGB primaries instead, working within the gamut of the device to match colors. Second, while there are many clever ways to let observers mix colors (via knobs, sliders, or even by tilting the device), it is hard to give observers the same level of control seen with physical knobs, but that is a bit of a weak argument; the larger problem is that given a reference color and a few simple controls, observers are likely to end up mixing colors in a repetitious way when more random results would be expected within the range of indiscernible difference. This could be somewhat averted by techniques such as switching the order of the mixer controls between tests. Ultimately, if the end goal of such a test is to find colors which observers find indistinguishable from a reference color then it seems it might be simpler

and faster to generate colors close to the reference and ask the observer if they can tell the difference. 


\section{Color Discrimination}

When exploring the idea of mimicking a color discrimination test inspired by the Farnsworth Munsell 100 Hue Test (FM 100 Hue Test) [8] it quickly became apparent that this test, while useful for testing personal color vision discrimination and possibly drawing correlations between color discrimination abilities and personal/experimental attributes (gender, age, ethnicity, light source, viewing angle etc), was not useful for contributing to the model of the world average observer. The test itself is designed, "to separate persons with normal color vision into classes of superior, average and low color discrimination and to measure the zones of color confusion of color defective people" [8] and can score in such a way because it prescribes a correct color ordering. Ultimately this type of test doesn't help us understand colors within just noticeable difference (at least for

superior color observers) and likely cannot help us understand discernment of larger color differences. This said, a color discrimination test similar to the FM 100 Hue Test is likely a great measure to use before experiments to check for color deficiencies in a known and measured way. There may be other more useful ways to use color discrimination tests to gather the data needed to improve the average world observer model, but this type of test on a mobile device is probably too time consuming for the kind of results it delivers.

\section{Contrast Matching}

Color matching is used in many recent color discrimination studies [39, 36]. The basic premise is that while there is a yes/no answer to the question of can an observer tell if there is a discernible difference between two colors, it is harder to ask an observer how different two colors that are clearly different are. The usual 
strategy is to provide a reference pair of greyscale colors and to ask the observer if the difference between a provided color pair of colors is greater than, less than, or equal to that of the greyscale reference pair. Another strategy is to give the observer a set of greyscale pair samples and ask them to choose the one with the closest match in difference to a provided reference color pair.

It is my opinion that this is currently the best method for measuring medium to large color difference discernment, but it is an awkward test to give comparing greyscale samples to color ones and the test's origin seems to be driven by precomputing technology. A possible digital variation of this test that may be easier on the observer by allowing the observer to adjust the contrast between the colors in the greyscale pair until the observer is satisfied that its difference matches that of the colored pair. This comparison still seems awkward and hard for users to judge.

\section{Color Difference Discernment}

A color difference discernment test can be carried out in a number of forms but in its simplest form tests if a user can discern the difference between two colors or not. This is obviously only useful for testing the threshold of just noticeable difference around a reference color, but can be modified to test for more. This simple, binary test seems to serve as a modern replacement for color mix matching tests, but as it is quickly becomes less than useful with color pairs outside of the just noticeable and small difference range. 


\subsection{The Proof of Concept}

Cheaper, quicker color difference evaluation using digital displays has largely been ignored for the last couple decades for a couple of good reasons [17, 20, 39]. However, despite their shortcomings and considering recent improvements we cannot continue to afford ignoring digital alternatives. Mobile devices with ever improving color reproduction [26] and a wealth of sensors provide a relatively easy means to gather new, dense experimental data at a scale researchers like MacAdam once called impossible.

How digitally gathered datasets compare to physical ones has only begun to be explored; it is currently unclear if the results of digital tests (using colors that are in gamut) match up to more established physical ones [17, 39]. It is also likely that this form of data gathering will be very noisy from inter-observer variability [39], but with enough observers, tests, and previously unrecorded experimental data, we may be able to mitigate these problems enough to make meaningful advances. It is possible that in this trade-off of control for scale we will not be able to advance our understanding of perception further than we already have; regardless, it is a worthwhile endeavour to explore the wealth of new experimental opportunities crowdsourced color perception experimentation on mobile devices provides, even if only to prove that it is not yet possible and to help understand why.

After surveying the advantages and shortcomings of existing color tests, it is clear that the field could benefit from new creative ways to test the degrees of color difference perception. From this survey and examination of the CIE Guidelines I propose a new, arguably more robust method of experimentation based on the statistical method of paired comparisons, to help cope with the additional noise 
that can come from digital and crowd sourced testing, and to help highlight interand intra-observer variability. This variation of a color difference discernment test uses a center reference color and two comparison colors placed on either side, and asks the observer to choose which color is closest to the reference (Figure 3.1). This kind of test is unique in some ways, but is related to existing tests [36, 19, 9]. Additionally, by conducting this test in a crowd sourced manner via mobile devices, the test itself can be iterated, and performed more rapidly at a scale never before tested. In addition, this method of experimentation is highly extensible and adaptable to changing experimental conditions, evolving data sets, and new hypotheses.

While each individual comparison the observer makes is a discrete test, the strength of this experimental method relies on the information that can be gleaned from a completed test session. The combination of information from each individual comparison test, along with what can be inferred from a session of comparison tests (plus additional information provided by the mobile device being tested on regarding environmental and testing conditions) allows us to identify poor judges, poor testing environments (environment, device, etc), and even poorly designed tests to a degree.

By testing the same observer with the same color pair multiple times, testing multiple observers with the same test, and overlapping sets of tested colors with other observers, we can potentially stitch together a larger continuous set of results. After we have a large pool of test results we can filter and purify noise out of the data and draw new insights on inter- and intra-observer variability. Through crowdsourced testing, we essentially observe if the variables were controlled (filtering accordingly) rather than trying to control the variables. 


\section{Figure 3.1: A screenshot of the proof-of-concept app}

\subsubsection{Experiment Specifics}

The number of paired comparisons tests in one test session is determined by the following formula where $n$ is the number of colors being compared to the reference center color and $m$ is a redundancy multiplier (In a paired comparisons test we throw out tests of an object against itself, which is why we subtract $n$. We divide by 2 as to not double count the same paired comparison with a flipped object ordering.).

$$
\text { Number of tests in a session }=m * \frac{\left(n^{2}-n\right)}{2}
$$

As an example (Figure 3.2), imagine we generate a test session where we want to test a reference red color against three slightly different red colors $(A, B, C)$. First we generate the pairs the reference will be compared to in each test such that color A will be tested against B, A against C, B against C, and so on, skipping 
testing colors against themselves. We then generate the same pair tests $m$ more times to ensure that an observer will see the same pair multiple times to test for consistency. An observer that disagrees with their own judgements enough will have their results thrown out unless a large enough portion of the population also disagrees with themselves on the same pair, which would mean those two colors are hard to discern from one another. After all the pairs are generated, their order (including the order of the colors within each pair) is randomized. This randomization can happen for each observer or just once before the test is given to multiple participants.

After generating a set of tests, the observer is shown each pair of colors with the reference in the center and asked to choose which color in the pair is closest to the reference color. Time is not limited or recorded for each test, but could be. Each of the three color patches are equally-sized and positioned with equal space between them to reduce the known illusory impact of size [34] and distance 1 . The color squares are placed on a neutral grey background in an attempt to reduce the bias of the chromatic and lightness crispening effects (this effect causes a measurable increase in the perception of difference between two colors when the surrounding color is close to the samples in lightness or chroma); most researchers attempt to minimize this bias by consistently using a measured neutral grey [34, 13]. Between making color selections, the color patches (excluding the reference) are briefly replaced with the same neutral grey used as the background to mitigate a burn-in effect (this brief break likely is not long enough to counteract the illusory effects of staring at a sample for a long period of time, but could be adjusted accordingly and is better than nothing).

\footnotetext{
${ }^{1}$ The farther apart two colors are the more similar they appear. When two colors are sideby-side with no separation, differences are especially apparent 34
} 


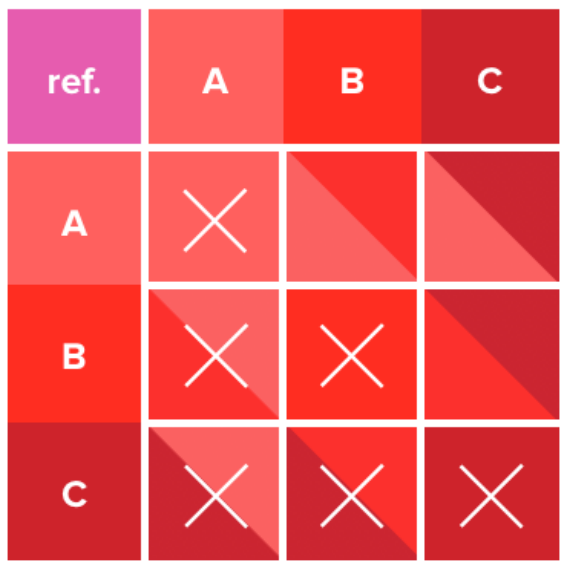

create pairs, throw

out duplicates $(n=3)$

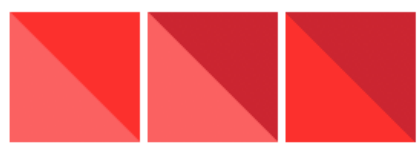

multiply tests for redundancy $(m=4)$
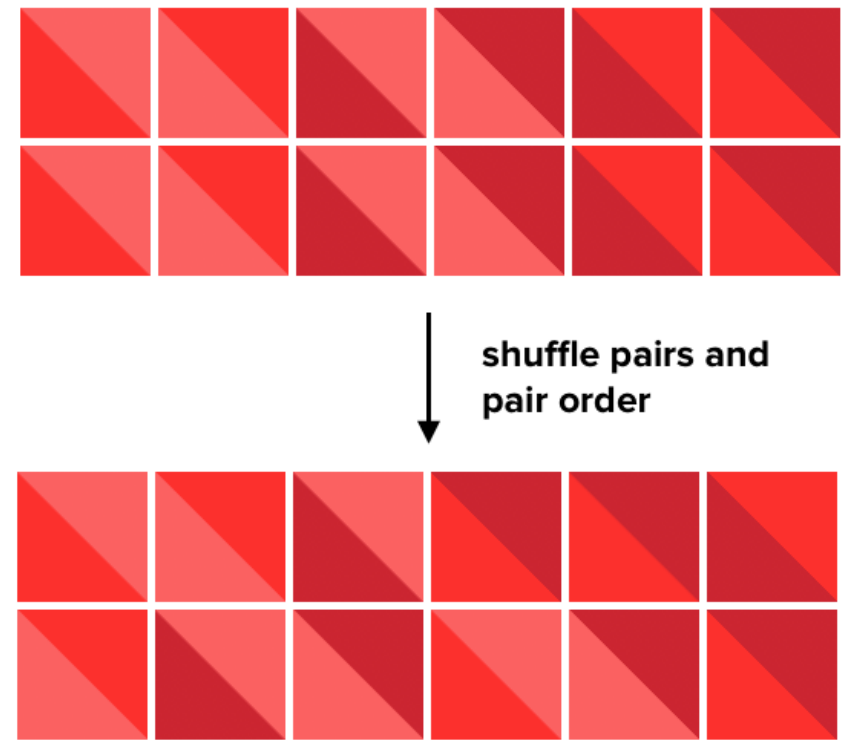

shuffle pairs and pair order
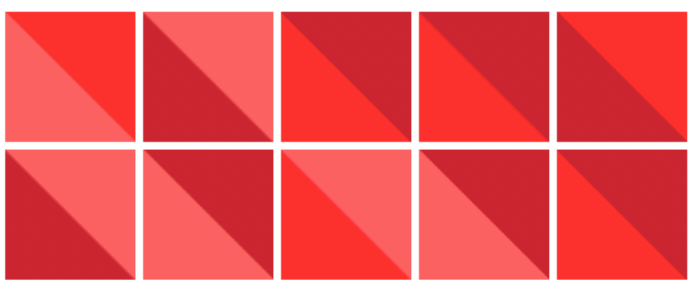

Figure 3.2: An example of generating the paired comparisons tests for a test session using 3 comparison colors (reds) and 1 reference color (purple) 
While each test is relatively quick, the number of tests required for each additional color increases exponentially. For example, using our Equation 3.1. testing 3 colors with triple redundancy takes 18 pair tests, while 6 colors balloons to 90 , which can be reduced to 60 if only double redundancy is used. This, however, is not impeding since crowdsourced test sessions can be organized such that they are repeated with similar judges/environments, or even partially overlap to connect smaller datasets into more meaningful larger ones.

\subsubsection{Paired Comparisons}

Thurstones Law of Comparative Judgment and the Method of Paired Comparisons [38] is a widely cited method of statistical analysis for quantifying and ranking preferences of things that are typically hard to quantify and directly compare to one another. Paired Comparisons makes it relatively easy to rank things like foods, job options, election candidates, etc. The method itself describes an experiment design that can be analyzed by a set of statistical methods to rank its inputs and analyze the validity of the results based on the consistencies of observers. The basic premise relies on putting every option to be compared against one another in the style similar to a double round-robin tournament (see the top illustration in Figure 3.2. The observer is then presented one pair at the time to choose between based on the question being asked. For each pair the winner is assigned a point; the score of each comparison is recorded separately for further analysis. Once all pairs have been tested, scores are tallied per row, representing all of the comparisons for which that object was chosen (in Figure 3.2 , if for the two tests in row $\mathrm{A}, \mathrm{A}$ was chosen in the first pair, and $\mathrm{C}$ was chosen in the second pair, the score for row A would be 1). These row totals can then be used to rank the choices. There are two validity checks to run before using this data however. 


\section{Using Paired Comparisons Data}

One of the benefits of Paired Comparisons is how it inherently captures judge consistency information. One way to check for consistency is by looking for the number of circular triads an observer makes. Triads are formed when votes contradict the transitive property. If someone prefers $\mathrm{A}$ over $\mathrm{B}(A>B)$ in a paired comparison and then $\mathrm{B}$ over $\mathrm{C}(B>C)$, we can say that that person should prefer A over $\mathrm{C}(A>B>C \equiv A>C)$. In the case of two choices, a disagreement might just signify a difference between the three that is within just noticeable difference (in other words are perceptually the same), but in the case that a loop is formed $(A>B, B>C, C>A)$ there is a disagreement and a circular triad is present. These triads occur because either the objects being compared are imperceptibly different to the observer either due to personal perception, environmental conditions, or just poor test taking 2 .

Formulas are available for calculating total number of triads in a set of paired comparisons for an observer and for creating a coefficient of consistency by comparing found triads with total possible triads. When a judge has a consistency coefficient that is anomalous with the group, then we can identify that they may have lower perception of differences relative to the group or that their testing environment was relatively faulty. When a measured majority of judges have poor consistency coefficients, it might be a sign of a bad test, or imperceptible differences. Additionally, if a majority in the whole population of judges forms the same triads there may be an imperceptibility of difference between those objects.

\footnotetext{
${ }^{2}$ All of these assumptions can be more-or-less confirmed by comparing results to the global results and to recorded environmental variables such as light, device movement, etc.
} 


\subsubsection{Following the CIE Guidelines}

The CIE Guidelines were kept in mind while designing this experimental method, with considerations given to the fact that guidelines were published in 1978, long before color experiments using digital displays could be conducted. Largely this experimental method hopes to follow Steps 1 and 2 to be a means of providing data for future research following Steps 3 and 4.

\section{Step 1}

Being a digital experiment, almost any color center can be tested, granted it and the range of colors around it being tested fall into the gamut of the device space. It is important that the experiment be run on a device with a known gamut so that the mapping of device-independent Lab colors to device-dependent RGB is accurate. In our testing Apple's iPhone 5 and 5 s were used ${ }^{3}$, both which have a known and consistent sRGB gamut. As for the test itself, we determined that a visual colorimeter or color-difference simulator testing approach did not make sense considering the medium, and that a test that modeled object color experiments was more apt. Typically, the limiting factor of object color testing has been having a limited number of samples, creating them, making sure they do not degrade, avoiding metamerism, and making sure they are not fluorescent 4 . Almost all of these concerns essentially become non-issues on a digital device with a known, well-controlled gamut5. This is not to say there are not other concerns with digital reproduction of color (discussed more later). Running an experiment

\footnotetext{
${ }^{3}$ Pilot study experiments were run exclusively on an iPhone $5 \mathrm{~s}$

${ }^{4}$ Fluorescent in this sense means emitting light from absorbing light (for example a substance that glows blue under UV light in normal daylight containing invisible UV light).

${ }^{5}$ However even quality displays with faithful consistent color reproduction across devices can degrade different over time or have defects off the factory line resulting from inadequate quality control.
} 
like this in a digital crowd sourced manner makes the recommended experiment repeat and observer counts (of at least 4 and 20 respectively) suggested by the standard, trivial to meet.

The paired comparisons style experiment we use falls under the suggested visual scaling method and the raw data gathered should be comparable to other similar studies. It should be noted that we did test experiments that modeled after the other two suggestions of the guidelines (the Rich-Billmeyer-Howe method and comparison with a constant color difference method), but determined the paired comparisons method to produce more robust and versatile data, and to be the easiest for observers to understand.

\section{Step 2}

Step 2 is about testing the various parameters that are known to affect color difference perception 5 , By the nature of our experiment being digital and crowd sourced, testing the effects of all but two of the parameters described (texture of samples, and monocular vs. binocular observation) should be relatively simple. By targeting observers that have already taken a pilot test with an identical test with one tweaked parameter, we should be able to build an understanding of how these parameters effect perception.

Simple experimental attributes like sample size, sample separation, color of surround, duration of observation, and size of $\Delta E$ can all be easily tweaked and tested. Furthermore with crowdsourcing, it is possible to test the fine-grained effects of slight experimental parameter changes by automatically adjusting parameters and generating new tests to send out, based on currently received results.

\footnotetext{
${ }^{6}$ The quantified degree to which these parameters effect perception is largely disputed and unknown 9 .
} 
For example if we want to test the size of samples we could send out two modified tests to our $n$ observers who have already seen the original, one with a slightly smaller sample size, and one with a slightly larger one; based on the results we get back, we could then choose to investigate (manually or automatically) some finer grain of in-between sizes where perception is most contentious to help quantify how that particular parameter affects perception.

Parameters like illumination level and luminance level are more questionable to test on mobile devices in uncontrolled environments, but still can be tested to a degree to possibly provide new insights. By controlling the brightness of the device screen we can test how illumination level affects perception, although it must be noted that by the nature of being displayed on a screen, the samples are emitting light rather than reflecting light from an illumination source. Luminance factor (the ratio of luminance of a sample compared to a perfect diffuser under the same lighting conditions) is probably near impossible to test, but a similar variable we can study is the affect of environmental light at the time of testing. By using either light sensors built into devices (usually used to adjust screen brightness automatically), or front-facing cameras, we can measure the intensity of the light in the testing environment to a degree. This information can later be correlated to changes in perception, or can be used to filter test results by throwing out tests that either are outside of a certain range or that fluctuated too much during testing.

\subsection{Pilot Study}

The first purpose of the pilot study was to build and test a simple iteration of the application (app) to determine best usability practices and platform lim- 
itations. The second purpose was to use the built app to collect a relatively small, but statistically significant, dataset in order to estimate the rough level of variability in the data and the scale of data that might be needed in a full study.

\subsubsection{App details}

The app was built for iOS in order to target Apple's iPhone 5 and 5s; both devices use a reliable, much studied sRGB display [26, 16]. Sizing and spacing of the samples was chosen somewhat arbitrarily and need to be studied more. When choosing sizing and spacing, consideration was given to providing a decent margin around the samples while making the samples themselves square and as large as possible. Careful thought was given as to not use borders or markings near the samples. The small visual indicators and labels used on the screen were colored in greys and appropriately distanced from the samples. When possible, subtle animations were used instead of colored markings to convey which areas of the screen were tappable.

The app was built to set the device's backlight brightness to $100 \%$ upon launch and to set it back to $100 \%$ if the observer changed it during the test. Whenever the brightness changed, a small explanatory notification was shown at the top of the screen. Every test used a random test/pair ordering. For posterity and future studies, this random order of the tests and the colors in the pairs was recorded for each observer. The time the test was completed was also recorded. It would have been possible to record the time taken for each pair and the whole test session, but this information was not recorded or used for our pilot study. All color data was recorded as the intended Lab color values as well as the sRGB values after translation. 


\subsubsection{The Colors Chosen}

For the pilot study, we used the red specified in the CIE Guidelines [9]. In addition to complying to the standard we decided to test this red because it is used and cited in existing research about color perception testing on LCD displays or by using object colors and the method of paired comparisons [36, 39].

To choose the colors we would compare to the red we looked at the 70 colors tested against the red color center in the previously mentioned LCD research experiment. We sorted these colors according to the CIEDE2000 distance formula and then chose 5 evenly spaced colors along the distribution. Due to an experimental oversight, the Lab values for the standard red specified by the CIE were used instead of the slightly different Lab values used in the LCD experiment (The difference between the two being a CIEDE2000 distance of 1.5524). This CIE defined red was used consistently throughout the rest of the experiment as the color center. Since the 70 colors used in the LCD experiment were created by choosing 5 points along 14 equally distributed vectors of equal magnitude from the color center, an alternative testing approach would have been to test 5 colors along a single vector. Out of 5 sets of 70 colors tested in the LCD experiment, 9\% of the colors generated were determined to be identical when converted from Lab to RGB [39]; none of the colors used in our pilot study mapped to the identical RGB values.

\subsubsection{Statistical Verification}

The pilot study used eleven observers, consisting of students and professors of ages between 20 and 60 with a majority falling between 20 and 25 . Testing took place in various conditions with varying surounding light. All observers reported 
Table 3.1: Colors used in pilot study

\begin{tabular}{|c|c|c|c|c|c|c|}
\hline & & CIE Lab & & & sRGB & \\
\hline & $\mathrm{L}^{*}$ & $a^{*}$ & $b^{*}$ & $\mathrm{R}$ & G & B \\
\hline Ref. Red & 44.4 & 37.9 & 23.3 & 0.665814 & 0.298181 & 0.268118 \\
\hline Red 0 & 44.278000 & 37.709999 & 23.469000 & 0.663651 & 0.297694 & 0.265869 \\
\hline Red 1 & 44.306999 & 35.611000 & 23.422001 & 0.653566 & 0.306167 & 0.265891 \\
\hline Red 2 & 45.726002 & 37.209000 & 21.694000 & 0.675875 & 0.314403 & 0.290424 \\
\hline Red 3 & 46.313000 & 37.750999 & 21.889000 & 0.685503 & 0.318068 & 0.294655 \\
\hline Red 4 & 47.866001 & 36.321999 & 22.391001 & 0.696618 & 0.338833 & 0.305284 \\
\hline
\end{tabular}

being color normal (in a proper test this would need to be more explicitly tested via a test like the Ishihara plates or Farnsworth FM 100 or D-15 test). Five observers were female, and six were male.

Once we had the data for each observer we were able to sum the results of each row/color (method described in Paired Comparisons section) to see each observer's color ranks. This rank was essentially the result of 8 color pair choices. In this test, since the colors were chosen to be equally distributed along the 70 sorted colors (sorted in regard to their CIEDE2000 distance from the reference color) from the LCD research experiment, we would expect observers with perfect discernment to rank the colors in index order with an row rank score of 16 for red 0,12 for red 1,8 for red 2 and so on.

We then calculated the standard deviation of these ranks for each color across all observers. The resulting standard deviations and standard error (standard deviation normalized for the sample size), suggests that on average, observers only deviated from the average rank by one test for each color. This error is 
Table 3.2: Expected/Average Rank, and Standard Deviation/Error of Ranks per Color (row totals)

\begin{tabular}{lcccc} 
& Perfect Rank & Avg. Rank & Standard Deviation & Standard Error \\
\cline { 2 - 5 } red 0 & 16 & 14.82 & 1.168 & 0.352 \\
red 1 & 12 & 12.45 & 1.036 & 0.312 \\
red 2 & 8 & 7.09 & 1.375 & 0.415 \\
red 3 & 4 & 5.27 & 1.009 & 0.304 \\
red 4 & 0 & 0.36 & 0.924 & 0.27872
\end{tabular}

relatively low, but this pilot study employed a small sample size and only tested one color center with five colors that were relatively well-spaced in regard to CIEDE2000 distance. As a sanity check, this superficial analysis suggests that the test on a mobile device in various settings is a viable testing method to be explored further, especially when we consider that a more complex analysis of the data and error would allow us to filter noise and draw more complex insights of intra- and inter-observer variability.

Because of the variables discussed in previous sections, it is hard to determine how many observers would be necessary in a statistically relevant sample for a test of this nature. If we are able to detect a relatively balanced and mostly symmetric distribution of answers in a substantial pilot test, the Central Limit Theorem tells us that we will need approximately 30 observers per test to produce results in the $95 \%$ confidence range. Once we take into account the number of these tests necessary to cover all color centers along multiple vectors, overlap the tests, account for noise, and inter/intra-observer variability, the number will surely balloon, but not excessively for a crowdsourced experiment. 


\subsection{Future Work}

Unfortunately, executing a full test of the experimental methods described prior was outside of the scope of this thesis, however, we have thought through many of the advantages this type of crowdsourced mobile testing can capitalize on, along with known shortcomings and problems that will need to be overcome or taken into account.

\subsubsection{Crowdsourcing}

Crowdsourcing is not a typical method employed in academic experiments and has only recently begun to be seriously considered and used by researchers, largely due in part to advancing technology and the internet. With a sizeable portion of global population carrying highly accurate displays in their pockets, loaded with sensors, it is suddenly possible to poll a large swath of the population in a fraction of the time that it takes to get a single person into the lab. The potential of global crowdsourced experiments and research has only begun to be explored and understood for what it is worth. We hope that the previous proofof-concept and ideas presented here serve to encourage future color perception research that capitalizes on mobile crowdsourcing.

\section{Examples of Current Experimental Crowdsourcing}

Examples of successful crowdsourcing with the purpose of furthering scientific understanding reach back to the 1900s with The National Audubon Society's Christmas Bird Count. This experiment, which started at the turn of the century with 27 citizen scientists, had 71,531 citizen scientist participants in their world- 
wide 2012 winter count [32]. The iNaturalist iPhone app (a project that started as a UC Berkeley Masters final project) has a community of over 37,000 global citizen scientists as of May 1st, 2014 that help record biodiversity by contributing to a set of over half a million recorded observations [1]. In 2014, following the disappearance of a commercial jet over the Gulf of Thailand, a satellite company named DigitalGlobe opened a crowdsourced campaign that allowed anyone to pour through recent satellite images of the region to flag potential debris and clues [35].

These are all examples of crowdsourcing that tap engaged citizen scientists, rather than the average citizen, to collectively solve huge problems. Even though these projects only ostensibly help the participants, they are all backed by tens of thousands of volunteers. Considering the over 200,000 user sessions that contributed to popular web cartoonist Randall Munroe's color naming survey in 2010 [27] and the viral popularity suggested by the nearly 800,000 social shares of XRite's (parent company of Pantone) digital version of the Farnsworth Munsell 100 Hue Test [8], it is not hard to see how a color perception test distributed as a mobile app could potentially have a significant number of contributors.

\section{Sweetening the Deal}

Sometimes it is not easy to gain significant voluntary participation in crowdsourced experiments. In these cases, sometimes the way the data is collected has to be tailored to benefit the participant. The Track Your Happiness app, which asks participants questions about their happiness throughout the day, not only collects meaningful research data, but provides personal insights to participants along the way. The project began as a doctoral project in 2009 with 1,000 users

and has grown to have over 15,000 highly diverse participants filing a total of 
650,000 reports as of 2011 [40, 37]. The rate at which people return to use the Track Your Happiness app multiple times is a great indicator of how providing interesting feedback to participants can keep them engaged. It is not hard to imagine tweaking a test like ours to provide participants feedback on when they perform best, what colors they have the most trouble discerning, and perhaps a visualization of what colors they have been tested on and how much they have contributed to a global set of data, to make the experiment more engaging. With an engaged testing population, new tests can be continually created and sent down (automatically or manually created) to test new hypotheses, points of high variability, and experimental parameters such as color swatch size and spacing.

\section{Inadvertantly Contributing}

There are other potential methods of crowdsourcing such as following the reCAPTCHA model. In the reCAPTCHA model, participants online needing to prove their humanness do so by typing hard to recognize words and numbers, where one of the two is actually a scan/photograph of a book or house, thereby helping digitize text and annotate scanned literature [2]. In the reCAPTCHA model there isn't necessarily a correct answer for the second word or number, but when a public consensus is reached it can be considered the right transcription with high probability. Small color tests could be used alongside other reverse turning tests to slowly build up a large set of color perception data. This model is not as feasible for delivering lengthy tests or for tracking participants over time and across tests, and reCAPTCHAs typically are not seen on mobile devices where color conditions can be more easily known and controlled.

Another possibility is to pay participants online to participate in experiments through an online crowdsourcing marketplace like Amazon's Mechanical Turk ser- 
vice. Diversity of workers in these marketplaces is questionable with $57 \%$ coming from the US and $32 \%$ from India, and the population generally being younger and college educated [29]. Despite this, a more recent psychology study in 2011 found the workers to be "more demographically diverse than... standard Internet samples and... significantly more diverse than typical American college samples" [12]. The same study found that overall the data gathered "met or exceeded the psychometric standards associated with published research" [12]. Costs for administering medium length surveys on the order of around ten minutes generally vary, but appear to not cost more than $\$ 0.50$ [12, 29]. There are many other considerations to be had when building an experiment for deployment through Mechanical Turk, but it is nonetheless a cheap and promising crowdsourcing method. At the moment Mechanical Turk is a web only tool, making careful control of the devices used by observers relatively impractictal (or even impossible).

\subsubsection{Mobile as a Platform}

\section{Color Reproduction and Light}

Some of the obvious problems that come up when discussing using mobile devices for color testing is lacking color-reproduction, variance between displays, brightness settings, surrounding light conditions, viewing angle/position, and reflectance of the screen. While none of this is ideal, it is important that we strive for consistency in our data and where we cannot guarantee consistency, then we record all the pertinent data that we can.

As far as color reproduction goes, RGB screens even at their best cover no more than an estimated $35 \%$ of the gamut of human vision [10]; even with 6 primary colors they cannot cover more than $96 \%$ of human vision. In 1980, 
Michael R. Pointer created the Pointer's Gamut (see Figure 3.3), which sought to capture the range of reflected object colors that we see most day-to-day (based on 4089 samples), while leaving out more specular colors such as those seen in the sky [5]. This irregular gamut serves as proof that while our displays are not perfect, they arguably can capture the most important colors. By targeting devices with known gamuts that have measurably tight tolerances (such as Apple's iPhone 5 and 5s, which have a consistent sRGB gamut [16, 26]) we can assure that we are able to reproduce colors consistently across devices and within a significant portion of the Pointer's gamut.

By targeting a single, reliable device, we can take into account parameters like its maximum brightness, and reflectance of the screen to a degree. This naively ignores such things as backlight degradation, and cases or films that observers might have covering their screens. By recording or controlling the screen brightness we can help take the brightness variable into account, and by asking the user about films or cases before testing we presume we should be able to filter out compromised data relatively well.

Knowing surrounding light conditions can be tough; on the screen we can surround color swatches with neutral colors, but factors such as external light and even the color of the phone bezel could affect the results. Ambient light can be measured fairly well through device light sensors if availably 7 or more roughly via the front-facing camera.

While many tests restrict human vision by having the observer look through eyepieces or keep their head at a certain distance from the samples with samples

\footnotetext{
${ }^{7}$ The front-facing light sensor on iPhones is questionably accessible via a public API, but use of the API apparently results in Apple App Store rejection [4. It could still be possible to distribute such apps not through the App Store, but obviously it would be much harder to scale.
} 


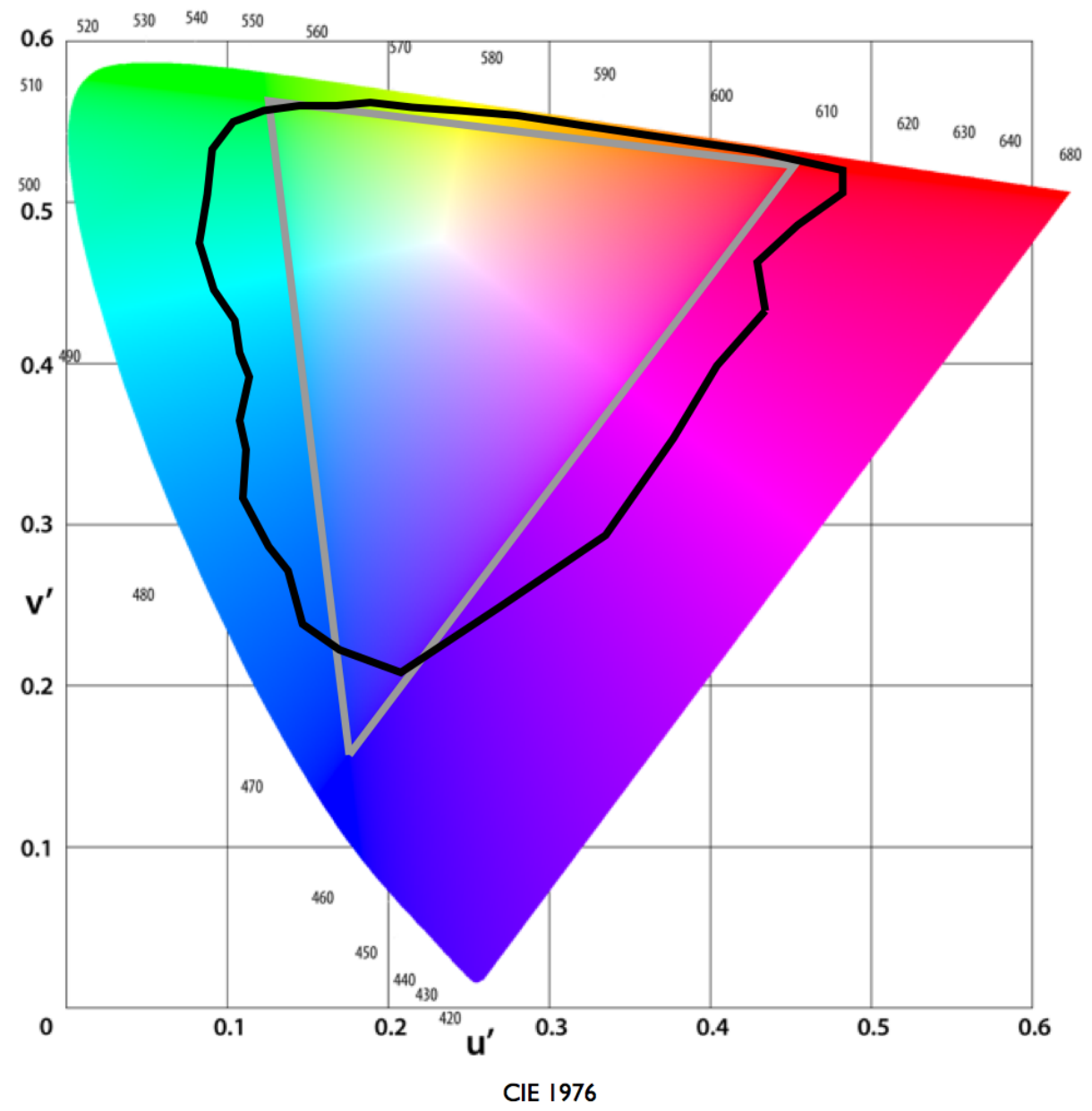

- Pointer's Gamut _ iPhone 5 Gamut

Figure 3.3: Pointer's gamut compared with the iPhone 5's [6] 
at a controlled angle relative to the eye and light source, a self-administered mobile test will be held unpredictably. While it is unclear how much relatively small movements or differing angles affect perception in a test like this, tilting the device at various angles, bringing it close or farther from the eyes while testing is likely to have an effect. While there's no easy way to determine the angle of the device relative to light sources in the room or to the observer's eyes, by recording accelerometer and gyroscopic data during testing, we can determine certain behaviors and levels of movement and filter tests accordingly.

\section{Capturing Additional Data}

An interesting example of the type of extra data we can easily gather with a mobile crowdsourced test is how long the observer takes to decide on a test choice. In our pilot test we observed observers taking significantly longer to decided between color pairs determined to be closer using CIEDE2000, while pairs that were farther away were answered relatively quickly. Going off of this assumption, it may be useful to collect the time taken to complete each test as a means to strengthen determining where observers contradicted themselves. Observers can explicitly contradict themselves through their pair choices; by analyzing this in tandem with the time taken to decide, we may be able to determine whether a pair decision was difficult because the colors were indistinguishable from one another, or because they were perceived as equally different from the center color (for example we might expect observers who regularly flipped their decision on a pair but answered quickly, to not see the difference between the pair colors, whereas observers that answered regularly, but took a relatively long time deciding on those pairs might have been more likely to have had difficulty choosing between the two because the colors seemed equally distant from the center). This 
timing data could also be used more simply to strengthen knowing which pairs observers contradicted themselves on (in actuality or though relatively lengthy deliberation).

It is known that the length staring at a sample can affect its perception as well [13] so by recording the time taken on each test we may be able to throw out tests where the observer could have been staring for long enough to be affected by an after-image. Additionally, by using timestamps and app data we can determine if the observer finished a test in one sitting or if they took their time between samples (making it more likely the environmental variables changed), and throw out or filter their data accordingly.

One of the biggest insights that may come from a study of this scale is a better understanding of inter-observer variability. Inter-observer variability is already thought to vary widely [19], therefore it may be that even our best model of a world-average observer does not represent us individually with high accuracy. There are innate differences between us all both neurobiological and cognitively that introduce variability into how we each perceive color. Physical changes such as macular degeneration [11] can further cause us to perceive color differently than others. According to Kuehni, even with the various ways we can fit formulas to observer data, "such a formula can be considered accurate for the mean observer... but it will represent the judgments of only about one third of real observers to within $\pm 20 \%$ of the calculated value" [19].

By asking observers in a widely crowdsourced test basic questions about age, sex, ethnicity, known vision impairments, and by administering basic color discrimination tests before our own paired comparisons testing, we may finally be able to model individual perception more accurately by realizing divisions in the population by which we can group observers. 


\section{CHAPTER 4}

\section{Conclusion}

Accurate understanding of color is a cornerstone of many industries, yet our current standards perform poorly, with an accuracy of predicting existing empirical data around 65 percent. While the physics of color remains measurable, the psychological experience and many of its intricacies is not. Despite this, we can continue to strengthen our models and understanding of perception with creative and concerted, empirical experimental efforts. In order to develop a better understanding of color perception, we need as Kuehni puts it, "a widely controlled and comprehensive effort" [18].

In understanding perception, we need to not only gather more data, but to study how the variables in our experiments affect perception. Additionally we need to better our understanding of the world-average observer and its pertinence to our models. These questions can be tested at a large scale while gathering color perception data through a crowdsourced mobile app.

Crowdsourced mobile testing as a method needs to be investigated further, but promises to be a potentially viable way to improve our understanding of the 
many facets of color perception at a scale, speed, and level of granularity not yet seen by the field at large. Ultimately, even if this method doesn't further the development of our color models and standards it provides an innovative use of paired comparisons, mobile crowdsourcing, and can shed new light on how experimental parameters and conditions affect perception.

We hope the ideas and proof-of-concept outlined here help to inspire and encourage future research which will bolster and reinforce our understanding of our most relied upon sense. Please do not hesitate to contact the author for any clarifications or questions ${ }^{1}$.

\footnotetext{
${ }^{1}$ ryanmcleod@gmail.com
} 


\section{BIBLIOGRAPHY}

[1] About inaturalist. http://www.inaturalist.org/pages/about.

[2] Google recaptcha. https://www.google.com/recaptcha.

[3] Hyperphysics. http://hyperphysics.phyastr.gsu.edu/hbase/vision/retina.html.

[4] iPhoneDevWiki. http://iphonedevwiki.net/

[5] The pointer's gamut. http://www.tftcentral.co.uk/articles/pointers_gamut.htm.

[6] TFT Central. http://www.tftcentral.co.uk/articles/pointers_gamut.htm.

[7] Wikimedia commons. https://commons.wikimedia.org.

[8] X-rite. http://www.xrite.com.

[9] Cie guidelines for coordinated research on colour-difference evaluation. COLOR research and application, 3(3), Fall 1978.

[10] Quantum dots unleash high-color-gamut performance in led-backlit displays. LEDs Magazine, October 2011. 
[11] A. D. Broadbent. Calculation from the original experimental data of the cie 1931 rgb standard observer spectral chromaticity co-ordinates and color matching functions, 2008.

[12] M. Buhrmester, T. Kwang, and S. D. Gosling. Amazons mechanical turk: A new source of inexpensive yet high-quality data? Perspectives on Psychological Science, 6(1), Jan. 2011.

[13] L. M. Crdenas. Evaluation of variability in visual assessment of small color differences. Master's thesis, North Carolina State University, 2009.

[14] P. A. Garcia, R. Huertas, M. Melgosa, and G. Cui. Measurement of the relationship between perceived and computed color differences. Optical Society of America, 24(7), July 2007.

[15] V. Greenwood. The humans with super human vision, June 2012. http://discovermagazine.com/2012/jul-aug/06-humans-withsuper-human-vision.

[16] C. Heinonen. The iphone 5 display: Thoroughly analyzed, September 2012. http://www . anandtech.com/show/6334/iphone-5-screenperformance.

[17] R. G. Kuehni. Color Space and Its Divisions: color order from antiquity to the present.

[18] R. G. Kuehni. Color difference formulas: An unsatisfactory state of affairs. COLOR Research and Applicaiton, 33(44), August 2008.

[19] R. G. Kuehni. Variability in estimation of suprathreshold small color differences. COLOR research and application, 34(5), October 2009. 
[20] R. G. Kuehni and A. Schwarz. Color Ordered: A Survey of Color Order Systems From Antiquity To the Present.

[21] I. Lissner and P. Urban. Improving color-difference formulas using visual data. 2010.

[22] I. Lissner and P. Urban. Upgrading color-difference formulas. Optical Society of America, 27(7), July 2010.

[23] M. R. Luo and B. Rigg. Chromaticity-discrimination ellipses for surface colors.

[24] D. L. MacAdam. Visual sensitivities to color differences in daylight. Optical Society of America, 32(5), May 1942.

[25] M. Meyer. Mark meyer photography. http://www.photo-mark.com.

[26] M. Meyer. iphone 5 color, September 2012. http://www. photomark.com/notes/2012/sep/25/iphone-5-color/

[27] R. Munroe. Color survey results, May 2010. http://blog.xkcd.com/2010/05/03/color-survey-results/.

[28] M. Nolle, M. Suda, and W. Boxleitner. H2si - a new perceptual colour space. In Digital Signal Processing (DSP) 2013 18th International Conference on, pages 1-6, July 2013.

[29] J. Ross, I. Irani, M. S. Silberman, A. Zaldivar, and B. Tomlinson. Who are the crowdworkers?: Shifting demographics in mechanical turk. In $C H I$ '10 Extended Abstracts on Human Factors in Computing Systems, CHI EA '10, pages 2863-2872, New York, NY, USA, 2010. ACM. http://doi.acm.org/10.1145/1753846.1753873. 
[30] S. M. Salvi, S. Akhtar, and Z. Currie. Ageing changes in the eye. 82(971), 2006.

[31] J. Schanda. Colorimetry: Understanding the CIE System.

Wiley-Interscience, August 2007.

[32] S. M. Scully. 11 reasons the christmas bird count rocks, Dec. 2013. http://mag.audubon.org/articles/conservation/11-reasonschristmas-bird-count-rocks.

[33] G. Sharma, W. Wu, E. N. Dalal, and M. U. Celik. Mathematical discontinuities in ciede2000 color difference computations. IST/SID Twelfth Color Imaging Conference.

[34] S. K. Shevell. The Science of Color. Elsevier, 2003.

[35] D. Stout. Crowd-sourcing campaign launched to find missing jet, Mar. 2014. http://time.com/19510/crowd-sourcing-campaignlaunched-to-find-missing-jet/.

[36] D. Strocka, A. Brockes, and W. Paffhausen. Influence of experimental parameters on the evaluation of color-difference ellipsoids. COLOR research and application, 8(3), Fall 1983.

[37] TED. Want to be happier? stay in the moment, Nov. 2012. http://www.ted.com/talks/matt_killingsworth_want_to_be_ happier_stay_in_the_moment/transcript.

[38] L. L. Thurstone. A law of comparative judgment. Psychological Review, 34:273-286, 1927. 
[39] P. Urban, M. Fedutina, and I. Lissner. Analyzing small suprathreshold differences of lcd-generated colors. Optical Society of America, 28(7), July 2011.

[40] J. Wortham. If youre happy and you know it tell your phone, July 2009. http://bits.blogs.nytimes.com/2009/07/29/if-youre-happyand-you-know-it-tell-your-phone.

[41] W. D. Wright. A re-determination of the trichromatic coefficients of the spectral colours. Transactions of the Optical Society, 30(4):141, 1929. 